\title{
Getting it done: dynamic incentives to complete a project*
}

\author{
Robin Mason ${ }^{\dagger} \quad$ Juuso Välimäki ${ }^{\ddagger}$
}

5 October 2014

\begin{abstract}
A principal wants an agent to complete a project. The agent undertakes unobservable effort, which affects in each period the probability that the project is completed. We characterise the contracts that the principal sets, with and without commitment. With full commitment, the contract involves the agent's value and wage declining over time, in order to give the agent incentives to exert effort. The best sequentially rational equilibrium for the principal also involves the agent's wage declining over time, while the worst sequentially rational equilibrium for the principal has a constant wage (and is in fact the unique stationary equilibrium). The best (weakly) renegotiation-proof equilibrium for the principal is achieved by a constant wage that maximizes the principals payoff, conditional on wages being constant. We compare these solutions to the efficient outcome.

Keywords: Principal-agent model, continuous time, moral hazard, project completion.
\end{abstract}

JEL classification: C73; D82; J31.

${ }^{*}$ We are grateful to Matti Liski, as well as numerous seminar participants, the editor GeorgeMarios Angeletos and referees for many helpful comments.

${ }^{\dagger}$ University of Exeter and CEPR. University of Exeter Business School, Streatham Court, Rennes Drive, Exeter EX4 4PU, UK, mason.robin@gmail.com. Robin Mason acknowledges financial support from the ESRC under Research Grant RES-062-23-0925.

${ }^{\ddagger}$ Aalto University School of Economics and HECER. Arkadiankatu 7, FI-00100 Helsinki, juuso.valimaki@aalto.fi. 


\section{Introduction}

In this paper, we consider a model in which a principal hires an agent to complete a project. The principal gains no benefit until the project is completed. Project completion and payments to the agent are the only verifiable events, since the principal cannot observe the agent's effort. The agent affects the probability of project completion by exerting effort. The principal's objective is to provide the agent with dynamic incentives to exert effort. We assume that the agent is risk neutral and subject to a limited liability constraint (in other words, the agent cannot make payments to the principal). Hence our dynamic results arise not from insurance motives, but from the optimal management of the agent's intertemporal surplus. The task of the paper is to analyse the dynamic incentives that arise in these settings and the contracts that are written as a result.

Dynamics matter for both sides. For the agent, its myopic incentives are to equate the marginal cost of effort with the marginal return. But if it fails to complete the project today, it has a further chance tomorrow. This continuation value means that the forward-looking agent reduces its current effort, substituting towards future effort. Hence this dynamic factor, all other things equal, tends to reduce the agent's effort towards project completion. Similarly, the principal's myopic incentives trade off the marginal costs of inducing greater agent effort (through higher payments) with the marginal benefits. But the principal also knows that the project can be completed tomorrow; all other things equal, this tends to lower the payment that the principal pays today for project completion. On the other hand, the principal also realises that the agent faces dynamic incentives; this factor, on its own, tends to increase the payment to the agent.

Our modelling approach allows us to resolve these different incentives to arrive at analytical conclusions. After characterising the efficient solution, we start by analysing the case in which the principal can commit to a full sequence of future wages. We show the way in which the principal resolves in its favour the trade-off between static incentives (which call for a high current wage) and dynamic incentives (which call for lower future wages): the agent's wage (and effort) falls over time. This is in stark contrast to the efficient solution, where effort is constant (when the principal and agent have the same discount rates); or increasing exponentially over time (when the principal is the more patient).

We then move on to analyse sequentially rational equilibria, in which the principal has no commitment ability but instead sets payments period-by-period. We show that the (unique) stationary sequentially rational equilibrium, in which the principal offers the same wage in each period and the agent takes the same action, yields the lowest equilibrium payoff to the principal. We characterise also the equilibrium yielding the highest payoff to the principal, linking it in an intuitive way to the full commitment solution. In this equilibrium, the agent's wage declines over time up to some deadline, after which it is constant (and positive). As in the full commitment solution, the declining payments give the 
agent an incentive to exert effort now rather than later. This is supported in equilibrium by the threat to revert to the worst equilibrium if the principal deviates. (Note that only deviations by the principal can be observed.) Finally, we consider (weakly) renegotiation-proof equilibria, of which there are many. We characterise the best equilibrium for the principal. It has a constant wage on the equilibrium path, and yields a higher payoff to the principal than the unique stationary equilibrium of the game.

As a real-world example to motivate our analysis, consider the on-going construction of a nuclear power plant in Finland. Finnish parliamentary approval for a fifth nuclear unit was granted in 2002. Areva, a French government-owned nuclear energy company, began construction in 2005 at Olkiluoto, Finland to build a European Pressurised Water Reactor (EPR) nuclear power plant. (See Thomas (2009) for further details.) The buyer (i.e., principal) is Teollisuuden Voima Oy (TVO), a company jointly owned by the Finnish government and Finnish power companies. The contract signed between TVO and Areva was a 'turnkey' contract: Areva is obliged to provide all work, materials and services necessary to enable TVO to 'turn the key' and take over an operational plant. In order to be operational, the finished plant must be approved by the Finnish Radiation and Nuclear Safety Authority. The agreement between the Finnish government and the Finnish power companies is such that TVO secures the output of the plant over its full life at prices set to fully cover whatever costs are incurred. In short, the Olkiluoto plant is a project that has no value to the principal before completion; for which completion is verifiable; and the value of which the agent cannot influence. These are all features of our model.

The project has had a troubled history. The original contract specified a completion date of 2009. From the start, problems arose so that after three years of construction, the plant was three years behind schedule and Areva was suffering severe losses. In December 2012, Areva estimated that the full cost of building the reactor would be about $\$ 8.5$ billion, or almost three times the delivery price of $\$ 3.2$ billion. TVO has not been able to give an estimate of when the project will be completed; some commentators have stated that the startup of the reactor could be delayed until at least 2018. ${ }^{1}$ While some of the problems appear to have been outside of Areva's control, others do not.

The contract signed between TVO and Areva involved a fixed price of $€ 3.2$ billion, with Areva bearing any costs in excess of that figure. The contract also included a penalty clause for late completion of $0.2 \%$ of the contract cost per week, up to $10 \%$ of cost. But in December 2008, TVO announced that Areva had filed a request for arbitration with the International Chamber of Commerce (ICC) concerning the delay and related costs. The case has yet to be settled, with Areva claiming $€ 2.7$ billion to cover additional costs, and TVO counter-claiming

\footnotetext{
${ }^{1}$ See http://uk.reuters.com/article/2014/02/28/tvo-olkiluoto-idUKL6N0LX3XQ20140228. Link accessible on 12 March 2014.
} 
$€ 1.8$ billion in compensation for construction delays. ${ }^{2}$

Our theoretical model shows general features of the optimal contract for the principal (in the example above, TVO) in different cases depending on the principal's ability to commit to a contract at the outset. In the two best cases for the principal - the full commitment solution, and the best sequentially rational equilibrium - the contract involves a completion payment that declines over time. This feature matches the original contract agreed between TVO and Areva, with the penalty clause for delay serving to reduce the completion payment made to the agent. An unfortunate consequence of such decreasing reward schemes is that the agent has less and less incentive to complete the task. In the ArevaTVO case, this is reflected in the recent statement of the Finnish Minister of the Environment: "The expected time to completion has never been this long during the project". Our model predicts that such outcomes with sub-optimal delays can arise on the equilibrium path with positive probability. Another consequence is that it is vulnerable to renegotiation. The legal manoeuvres by AREVA to re-interpret the contract could be viewed in this light.

\section{$1.1 \quad$ Literature review}

Our work is most closely related to Bergemann and Hege (1998), (2005). In these papers, the equilibrium contract involves a declining wage paid to the agent. But these papers involve learning - that is, beliefs that are updated over time; and hence the dynamics are driven by non-stationary beliefs. In contrast, the equilibrium contract in our model can exhibit dynamics (depending on the equilibrium), despite there being no learning, and the basic environment being stationary. By allowing for a convex cost of effort for the agent, our model allows a richer set of dynamic issues to arise. Our analysis of the best sequentially rational contracting and the best weakly renegotiation-proof equilibria yield insights that are not present in a model with binary effort choice (or equivalently, with a linear cost of effort), which has been assumed in much of the previous literature.

More broadly, our work is related to the literature on dynamic moral hazard problems: particularly the more recent work on continuous-time models. This literature has demonstrated in considerable generality the benefits to the principal of being able to condition contracts on the intertemporal performance of the agent. By doing so, the principal can relax the agent's incentive compatibility constraints; see, for example, Malcomson and Spinnewyn (1988) and Laffont and Martimort (2002). A number of recent papers (e.g., Sannikov (2007), Sannikov (2008), Willams (2006), Biais et al. (2007) and Biais et al. (2010)) have analysed principal-agent problems in continuous time. For example, in Sannikov (2008), an agent controls the drift of a diffusion process, the realisation of which in each period affects the principal's payoff. When the agent's action is unob-

\footnotetext{
${ }^{2}$ See http://uk.reuters.com/article/2014/02/28/tvo-olkiluoto-idUKL6NOLX3XQ20140228. Link accessible on 12 March 2014.
} 
served, Sannikov characterises the optimal contract quite generally, in terms of the drift and volatility of the agent's continuation value in the contract.

There are two main differences between this paper and e.g., Sannikov (2008) First, we concentrate on project completion. We think this case is of independent interest for a number of different economic applications. But we also think that our setting, while less general in some respects than Sannikov's, serves to make very clear the intertemporal incentives at work. In Sannikov's models, incentives may be back or front loaded: the agent's value can drift either upward or downward. In specific equilibria of interest in our model (the full commitment solution, and the best sequentially rational equilibrium for the principal), the agent's value drifts in only one direction: downwards. This is a direct consequence of the project completion setting. Secondly, as a result of focussing on project completion, we are able analyse a range of equilibria - not just the full commitment case studied by Sannikov - identifying key differences between them.

The results in the full commitment solution of our model are reminiscent of those in papers (such as Shavell and Weiss (1979) and Hopenhayn and Nicolini (1997)) that look at unemployment insurance. In these papers, a government must make payments to an unemployed worker to provide a minimum level of expected discounted utility to the worker. The worker can exert effort to find a job; the government wants to minimise the total cost of providing unemployment insurance. Shavell and Weiss (1979) show that the optimal benefit payments to the unemployed worker should decrease over time. Hopenhayn and Nicolini (1997) establish that the government can improve things by imposing a tax on the individual when it finds work.

Some aspects of our analysis are similar: for example, that the principal's optimal payment under full commitment decreases over time (c.f., decreasing unemployment benefits over time). The economic forces at work are different, of course. In the unemployment insurance papers, the need to smooth over time the consumption of the risk-averse worker constrains the incentives that can be offered through unemployment benefits. In this paper, the risk neutral agent smooths its effort over time; the principal sets a declining wage to counteract this incentive. But other aspects of our analysis are quite different. First, we go beyond the unemployment insurance papers by analysing the full commitment solution for all possible values of the discount rates of the principal and agent. This shows the importance of relative levels of patience in determining intertemporal incentives. Secondly, we provide a full characterisation of the set of sequentially rational equilibria, showing the benefits of commitment for the principal.

The rest of the paper is structured as follows. Section 2 lays out the basic model. Section 3 analyses the efficient benchmark where there is no need for the principal to hire an agent, and hence there is no moral hazard problem. Section 4 considers the case with moral hazard, in which the principal has full 
commitment power. Section 5 examines the opposite case in which the principal has no commitment power, analysing the sequentially rational equilibria of the game. Our overall conclusions are stated in Section 6. An Appendix contains longer proofs.

\section{The model}

We consider the continuous-time limit of a discrete time model where an agent must exert effort in any period in order to have a positive probability of success in a project. We assume that the effort choices of the agent are unobservable but the success of the project is verifiable; hence payments can be contingent only on the event of success or no success.

The principal and the agent are risk neutral. The agent is credit constrained so that payments from the principal to the agent must be non-negative in all periods; otherwise the solution to the contracting problem would be trivial: sell the project to the agent. (In fact, the agent could be allowed to be risk averse. The key assumption for our analysis is that the agent's value from contracting is positive, which here is a result of limited liability.) Successful completion of the project is worth $v \geq 0$ to the principal. The principal and the agent have respective discount rates of $r_{P}$ and $r_{A}$.

When the agent exerts effort $a$ within a time interval of (infinitesimal) length $\Delta$, the probability of a success is $a \Delta$. Hence we suppose that project completion follows a Poisson process in which the agent controls the arrival rate through her choice of effort. The cost of such effort is $c(a) \Delta$. We make the following assumption about the cost function.

Assumption 1 1. $c^{\prime}(a)>0, c^{\prime \prime}(a)>0$ and $c^{\prime \prime \prime}(a) \geq 0$ for all $a \geq 0 .^{3}$

2. $c(0)=0$ and $\lim _{a \rightarrow \infty} c^{\prime}(a)=\infty$.

Part 1 of the assumption is fairly standard, requiring the cost function to be increasing, convex and (even stronger) have a positive third derivative. The main substance of part 2 is to rule out fixed costs, so that the agent can ensure zero costs per period by exerting no effort. The assumption is satisfied e.g., for quadratic costs: $c(a)=\gamma a^{2}$, where $\gamma>0$.

We now describe the dynamic game between the principal and the agent. At the beginning of each period $t$, the principal promises to pay $w_{t}^{s}$ if the project is completed and $w_{t}^{f}$ if it remains incomplete. All past promises by the principal are observable but, as previously stated, the agent's effort choices are unobservable. Since the game ends once the project is completed (and this is verifiable), the only relevant histories in the game are those conditional on the project not being completed. We denote a history up to period $t$ by $h^{t} \equiv\left(w_{0}^{s}, w_{0}^{f}, \ldots, w_{t-1}^{s}, w_{t-1}^{f}\right)$.

\footnotetext{
${ }^{3} \mathrm{As}$ is often the case for clear comparative statics with dynamics or with uncertainty, we require the condition $c^{\prime \prime \prime} \geq 0$.
} 
The set of histories up to period $t$ is denoted by $H^{t}$. The set of all histories of all lengths is $H \equiv \cup_{t} H^{t}$. A wage setting strategy $\mathbf{w}$ for the principal is a pair of functions $\left(\mathbf{w}^{s}, \mathbf{w}^{f}\right)$ where $\mathbf{w}^{s}: H \rightarrow \mathbb{R}$ and $\mathbf{w}^{f}: H \rightarrow \mathbb{R}$. A continuation wage setting strategy after history $h^{t}$ is denoted by $\left.\mathbf{w}\right|_{h^{t}}$. Since the agent chooses his effort in period $t$ only after observing the promised wages, his strategy is denoted by $\mathbf{a}$ where ${ }^{4} \mathbf{a}: H \rightarrow \mathbb{R}$. A continuation strategy for the agent after history $h^{t}$ and current wage offer $w_{t}$ is denoted by $\left.\mathbf{a}\right|_{\left(h^{t}, w_{t}\right)}$.

Let $\tau$ denote the random number of time intervals of length $\Delta$ after which the project is completed. The expected payoffs for the principal and agent are then

$$
\begin{aligned}
V(\mathbf{w}, \mathbf{a}) \equiv & \mathbb{E}_{\tau}\left[\left(1-r_{P} \Delta\right)^{\tau}\left(v-w^{s}\left(h^{\tau}\right)\right)-\sum_{j=0}^{\tau-1}\left(1-r_{P} \Delta\right)^{j} w^{f}\left(h^{j}\right)\right] \\
= & \sum_{i=1}^{\infty}\left(\prod_{j=0}^{i-1}\left(1-a\left(h^{j}\right) \Delta\right)\right) \times \\
& {\left[\left(1-r_{P} \Delta\right)^{i} a\left(h^{i}\right) \Delta\left(v-w^{s}\left(h^{i}\right)\right)-\sum_{j=0}^{i-1}\left(1-r_{P} \Delta\right)^{j} w^{f}\left(h^{j}\right)\right], } \\
W(\mathbf{w}, \mathbf{a}) \equiv & \mathbb{E}_{\tau}\left[\left(1-r_{A} \Delta\right)^{\tau} w^{s}\left(h^{\tau}\right)-\sum_{j=0}^{\tau}\left(1-r_{A} \Delta\right)^{j} c\left(a\left(h^{j}\right)\right) \Delta+\sum_{j=0}^{\tau-1}\left(1-r_{A} \Delta\right)^{j} w^{f}\left(h^{j}\right)\right] \\
= & \sum_{i=0}^{\infty}\left(\prod_{j=0}^{i-1}\left(1-a\left(h^{j}\right) \Delta\right)\right) \times \\
& {\left[\left(1-r_{A} \Delta\right)^{i}\left(a\left(h^{i}\right) \Delta w^{s}\left(h^{i}\right)-c\left(a\left(h^{j}\right)\right) \Delta\right)+\sum_{j=0}^{i-1}\left(1-r_{A} \Delta\right)^{j} w^{f}\left(h^{j}\right)\right] . }
\end{aligned}
$$

While we have described the game in discrete time in order to make clear its dynamic structure, we shall throughout concentrate on the continuous-time limit of the game, as $\Delta \rightarrow 0$.

\section{The efficient solution}

We first consider the benchmark case in which there is no principal-agent problem i.e., a single individual chooses effort levels over time to maximise expected surplus. The problem will not be entirely standard, however: in order to reflect the analysis in the next Section, where the principal and the agent may have

\footnotetext{
${ }^{4}$ Since the stage game is in extensive form, and the principal moves first, the relevant history for the agent in period $t$ also contains $w_{t}$ and is thus $h^{t+1}$.
} 
different discount rates, we will apply the discount rate $r_{P}$ to the flow of benefits, and the rate $r_{A}$ to the flow of costs. We will also deal directly with the continuous-time limit for the single individual's problem, since in this optimal control problem, issues of timing within the period do not arise.

Consider a profile of actions $\{a(t)\}_{t=0}^{\infty}$. Given this profile, the probability that the project has not been completed by time $t$ is

$$
A(t) \equiv \int_{0}^{t} a(s) d s
$$

The single individual's problem is then to choose actions $\{a(t)\}_{t=0}^{\infty}$ to maximise

$$
\int_{0}^{\infty} \mathrm{e}^{-A(t)}\left[\mathrm{e}^{-r_{P} t} a(t) v-\mathrm{e}^{-r_{A} t} c(a(t))\right] d t
$$

subject to equation (3) and a non-negativity constraint on the effort level in each period i.e., $a(t) \geq 0$ for all $t$.

The optimization problem here can be considered as one where the principal and the agent contract in period zero and all future effort choices are contractible. (In order to maintain comparability to the equilibrium solutions presented in later Sections, there are no direct intertemporal transfers between the agent and the principal.) This results in a concave optimal control problem that can be analyzed by a routine application of Pontryagins maximum principle. Doing so shows that the single individual's optimal choice of action must satisfy the differential equation

$$
c^{\prime \prime}(a(t)) \dot{a}(t)=-r_{P} v \mathrm{e}^{\left(r_{A}-r_{P}\right) t}+\left(r_{A}+a(t)\right) c^{\prime}(a(t))-c(a(t))
$$

for all $t \geq 0$, and $a(t) \geq 0$. (Here, $\dot{a}(t)$ denotes the time derivative of $a(t)$.)

We shall see in the next Section that the principal-agent problem is nontrivial only when $r_{P} \leq r_{A}$; so we assume this for the rest of this Section. In order to characterise the solution, we first show in the next Lemma that any efficient solution must have effort non-decreasing over time.

Lemma 1 In any efficient solution, $\dot{a}(t) \geq 0$ for all $t$.

Proof. Suppose that at some time $t, \dot{a}(t)<0$. Since $r_{P} v \mathrm{e}^{\left(r_{A}-r_{P}\right) t}$ is increasing in $t$, and $\left(r_{A}+a\right) c^{\prime}(a)-c(a)$ is increasing in $a$ (from the assumed properties of the cost function), it must be that $\dot{a}(s)<0$ for all $s>t$. Moreover, since $-r_{P} v \mathrm{e}^{\left(r_{A}-r_{P}\right) t}$ is strictly negative, there must be some time $\tau$ at which $a(t)=0$ for all $t \geq \tau$. This cannot be optimal, however: such a path is strictly dominated by an alternative path that is the same at all times prior to $\tau$, but sets some strictly positive action for times subsequent to $\tau$.

With this Lemma, we can then describe the efficient choice of action. 
Proposition 1 When $r_{P}=r_{A}=r>0$, the efficient action is constant, and given by the solution to

$$
\left(r+a^{*}\right) c^{\prime}\left(a^{*}\right)-c\left(a^{*}\right)=r v .
$$

When $r_{P}<r_{A}$, the efficient action grows at least exponentially over time.

Proof. When $r_{P}=r_{A}=r>0$, equation (5) becomes

$$
c^{\prime \prime}(a(t)) \dot{a}(t)=-r v+(r+a(t)) c^{\prime}(a(t))-c(a(t)) .
$$

First note that there is clearly a unique solution to equation (6), since the lefthand side of the equation is increasing in $a$. Suppose now that $a(0)>a^{*}$. In this case, the differential equation implies that $\dot{a}(t)>0$ for all $t$, and hence that $a(t)$ grows without bound. This cannot be optimal. If $a(0)<a^{*}, \dot{a}(0)<0$, which cannot be optimal, by Lemma 1 . Hence $a(0)=a^{*}$, and consequently $a(t)=a^{*}$ for all $t$, in this case.

Now consider the case where $r_{P}<r_{A}$. Suppose that at any time $t, \dot{a}(t)=0$. Equation (5) implies that $\dot{a}(s)<0$ for all $s>t$, which cannot be optimal, by Lemma 1. Hence $\dot{a}(t)>0$ for all $t$. But then equation (5) implies that

$$
\left(r_{A}+a(t)\right) c^{\prime}(a(t))-c(a(t))>r_{P} v \mathrm{e}^{\left(r_{A}-r_{P}\right) t},
$$

which establishes the claim.

Some intuition is apparent from equation (6), if we re-write it $v=c^{\prime}(a)+$ $\left(a c^{\prime}(a)-c(a)\right) / r$. The static first-order condition is $v=c^{\prime}(a)$; the term $\left(a c^{\prime}(a)-\right.$ $c(a)) / r$ expresses the opportunity cost of effort today, when there is always the option of reducing current effort and completing the project in the future.

The dynamics of the efficient effort when the discount rates are not equal is of some interest. When costs are discounted more than benefits, effort is backloaded; the extent of the backloading depends on the relative discount factor $\mathrm{e}^{-\left(r_{A}-r_{P}\right) t}$. We shall see that this action profile is in sharp contrast to those that arise when a principal must contract with an agent.

We illustrate these solutions using a specific (quadratic) functional form for the cost function: $c(a)=\gamma a^{2}$; and specific parameter values: $v=\gamma=1$; $r_{p}=r_{A}=0.05$ in the equal discount rate case; and $r_{P}=0.02$ and $r_{A}=0.05$ in the unequal case. With this set-up, $a^{*}=0.1135$ and the efficient value, which is given by

$$
U^{*}=\frac{a^{*} v-c\left(a^{*}\right)}{r+a^{*}}
$$

is equal to $0.6154 . .^{5}$ The dynamics of the efficient effort when the discount rates

\footnotetext{
${ }^{5}$ In the quadratic case,

$$
a^{*}=\frac{-r+\sqrt{r^{2}+3 r v / \gamma}}{3}
$$

and

$$
U^{*}=\frac{(r+\sqrt{r(r+3 v / \gamma)})(r-\sqrt{r(r+3 v / \gamma)}+3 v / \gamma)}{3(2 r+\sqrt{r(r+3 v / \gamma)})}
$$
}




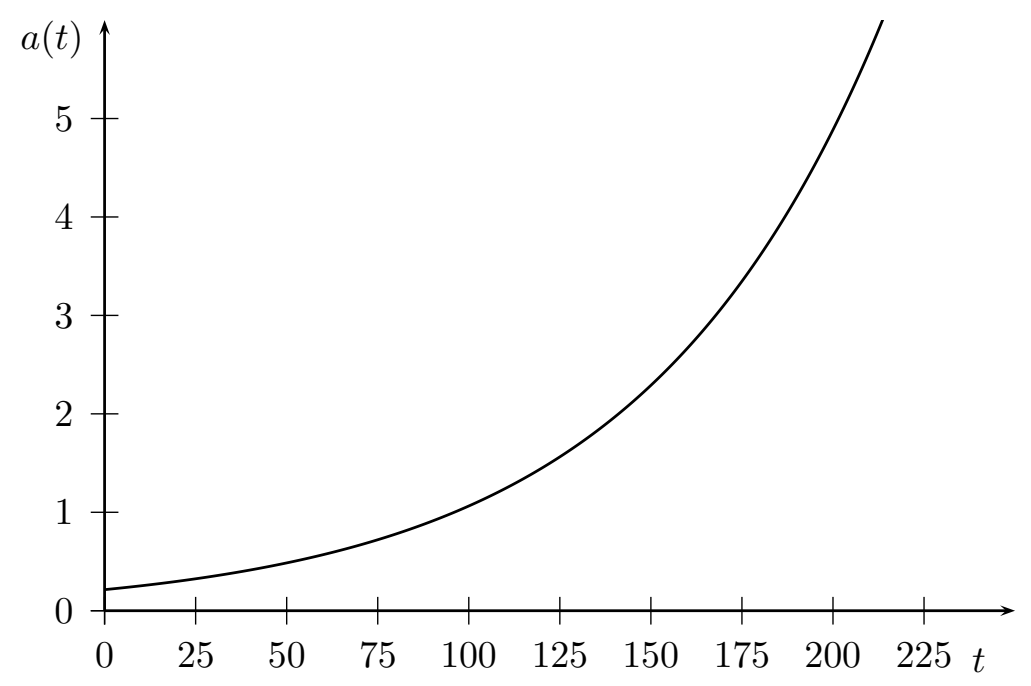

Figure 1: The efficient effort with unequal discount rates; $v=\gamma=1 ; r_{P}=$ $0.02, r_{A}=0.05$

are not equal are illustrated in figure 1 . The efficient value in this case is $0.7739{ }^{6}$

\section{Full commitment}

We now turn to the analysis of the principal-agent problem with moral hazard. We start by considering in this Section the case of full commitment, where the principal commits at the outset to a path of wages $\mathbf{w}\left(h^{t}\right)=w_{t}=\left(w_{t}^{s}, w_{t}^{f}\right)$ for all histories $h^{t} \in \cup_{t} h^{t}$. This corresponds to the Stackelberg solution of the sequential game where the principal first chooses a strategy once and for all at the beginning of the game, and the agent best-responds against this strategy. Most papers on dynamic incentive provision characterize the solution of this problem. We will derive this solution in some detail, since it will turn out that there is a close relation between the full commitment solution and the best sequentially rational equilibrium that construct in Section 5 .

We start the analysis by distinguishing between two cases. When the agent is more patient than the principal, the principal can induce the agent to take an arbitrarily high effort with an arbitrarily low cost. Because of the differences in the discount factors, a wage payment with a fixed present value to the agent is less costly to the principal (in present value terms) when the payment is delayed. So in this case, the principal can promise a high reward for success at negligible

\footnotetext{
${ }^{6}$ There is an analytical solution for the optimal effort in this case, involving Bessel functions. Numerical integration is used to calculated the value. The Matlab programme used for this and other calculations in the paper are available on request.
} 
cost through delayed payments. Hence in order to make the problem non-trivial, we assume from now on that $r_{A} \geq r_{P}$.

The following result will prove helpful in expressing the incentives that hold in the full commitment solution.

Proposition 2 In the full commitment solution, the principal sets the wage in the event that the project is not completed, $w_{t}^{f}$, at 0 for all $t$.

Proof. First note that it is obvious that any wages paid after a success can be paid immediately after that success: any delayed payment can be replaced by an immediate payment that is equivalent in net present value terms. Now consider an optimal wage path $\left\{\widehat{w}_{t}\right\}_{t=0}^{\infty}$ and fix the best response path of the agent $\left\{\widehat{a}_{t}\right\}_{t=0}^{\infty}$ against this wage path. There are two cases to consider, depending on whether the principal's value is (weakly) decreasing or increasing in the agent's choice of effort. Let $T \equiv\left\{t \mid V_{t}\right.$ is weakly decreasing in $a_{t}$ for $\left\{\widehat{w}_{t}\right\}_{t=0}^{\infty}$ and $\left.\left\{\widehat{a}_{s}\right\}_{s \neq t}\right\}$. Then for all $t \in T$, the principal increases her payoff by setting $\widetilde{w}_{t}^{f}=\widetilde{w}_{t}^{s}=0$. This increase is strict if $\widehat{w}_{t}^{f}>0$ for some $t \in T$.

Next consider $t \notin T$. We claim that the principal also sets optimally $\widehat{w}_{t}^{f}=0$. Suppose not i.e., suppose in some period $t \in T, \widehat{w}_{t}^{f}>0$. If $\widehat{w}_{t}^{s} \leq \widehat{w}_{t}^{f}$, then the optimal effort choice is 0 and the equilibrium payoff to the principal is negative and setting $w_{t}^{f}=w_{t}^{s}=0$ is a profitable deviation. Suppose next that $\widehat{w}_{t}^{s}>\widehat{w}_{t}^{f}>$ 0 . A deviation to $\widetilde{w}_{t}^{s}=\widehat{w}_{t}^{s}-\widehat{w}_{t}^{f}, \widetilde{w}_{t}^{f}=0$ leaves the incentives for optimal effort choice in period $t$ unchanged, noting that future wage offers by the principal are unaffected by this (since we are dealing with the full commitment case). This deviation reduces the wage payments of the principal by $\widehat{w}_{t}^{f}>0$ and hence is clearly profitable.

To see why Proposition 2 holds, suppose to the contrary that $w_{t}^{f}>0$ at some time $t$. The principal could then reduce $w_{t}^{s}$ and $w_{t}^{f}$ by the same amount, leaving all other wages constant. This has the effect of increasing effort in earlier stages while reducing the principals expected payment, and so must be better for the principal. For the remainder of this Section, we shall therefore just refer to the wage profile $\left\{w_{s}\right\}_{s=0}^{\infty}$ set by the principal, specifying the payment received by the agent depending on when the project is completed.

The next step for the analysis is to capture succinctly the incentives of the principal and the agent. First note that there is an implicit state variable in the game: whether the project has been completed or not. But since the game is over as soon as the project is completed, we do not emphasise this state variable. We can therefore consider time as the only state variable, and write the agent's problem in the following recursive way:

$$
W_{t}=\max _{a}\left\{a \Delta w_{t}-c(a) \Delta+(1-a \Delta)\left(1-r_{A} \Delta\right) W_{t+\Delta}\right\} .
$$

Here, $W_{t}$ is the agent's value function at time $t$, facing the wage schedule set by the principal, $\left\{w_{s}\right\}_{s=0}^{\infty}$. This value has three terms. The first is the expected flow 
payoff of the agent: with probability $a \Delta$ the project is completed and the agent receives the current wage $w_{t}$. The agent incurs a flow cost $c(a) \Delta$ regardless of whether his efforts are successful or not. Finally, if the project is not completed (with probability $1-a \Delta$ ), the game moves to the next period; the agent's value is then $W_{t+\Delta}$, which is discounted with the factor $1-r_{A} \Delta$.

Our next step is to consider the limit of the problem as $\Delta \rightarrow 0$. In this Section, we will take a heuristic approach to the continuous-time limits; in the Appendix, we take limits more rigorously, showing that they do indeed converge to the expressions derived here. When we take the limits, we denote the resulting variables at real time $t$ by $a(t), W(t)$ etc. ${ }^{7}$

Heuristically, we use a first-order Taylor series expansion and let $\Delta \rightarrow 0$ to arrive at a continuous-time Hamilton-Jacobi-Bellman (HJB) equation: $r_{A} W(t)=$ $\max _{a}\{a(w(t)-W(t))-c(a)+\dot{W}(t)\}$, where $\dot{W}(t) \equiv d W(t) / d t$. Since $c(\cdot)$ is strictly convex and any other term involving $a$ is linear in $a$, the first-order condition is necessary and sufficient for the agent's optimal action:

$$
w(t)=c^{\prime}(a(t))+W(t) .
$$

That is, the agent equates the marginal benefit from exerting effort (gaining the wage $w(t))$ with the marginal cost, which has two parts: the marginal cost of effort, $c^{\prime}(a)$, and the opportunity cost of succeeding, which is the foregone continuation value $W(t)$. Substituting into the HJB equation gives

$$
\dot{W}(t)=r_{A} W(t)-\left(a(t) c^{\prime}(a(t))-c(a(t))\right) .
$$

Equation (9) describes how the agent's value must evolve in equilibrium.

Using the first-order condition of the agent, we can write the principal's HJB equation in terms of choosing $a(t)$ as:

$$
V(t)=\max _{a}\left\{a(t)\left(v-w(t) d t+(1-a(t) d t)\left(1-r_{p} d t\right) V(t+d t)\right\} .\right.
$$

The interpretation of this equation is very similar to that of the agent's Bellman equation. Using equation (9) we have the following HJB equation for the principal:

$$
r_{P} V(t)=\max _{a}\left\{a(t)\left(v-V(t)-W(t)-c^{\prime}(a(t))\right)+\dot{V}(t)\right\} .
$$

When re-arranged, this becomes

$$
\dot{V}(t)=\left(r_{P}+a(t)\right) V(t)-a(t)\left(v-W(t)-c^{\prime}(a(t))\right) .
$$

Equation (10) describes how the principal's value must evolve in equilibrium.

\footnotetext{
${ }^{7}$ In other words, $a(t)=\lim _{\Delta \rightarrow 0} a_{k(\Delta, t)}$ along any convergent subsequence where we take $k(\Delta, t)=\min \left\{k^{\prime} \mid k^{\prime} \Delta \geq t\right\}$. Similarly for the other variables $w(t), W(t)$ and $V(t)$.
} 
We are now ready to write the full commitment problem in an optimal control formulation, where the objective is to maximize the principal's initial value, but with the continuation values of the principal and the agent as state variables: ${ }^{8}$

$$
\begin{gathered}
\max _{\{a(t), W(0)\}} V(0) \quad \text { subject to } \\
\dot{V}(t)=\left(r_{P}+a(t)\right) V(t)-a(t)\left(v-W(t)-c^{\prime}(a(t))\right), \\
\dot{W}(t)=r_{A} W(t)-\left(a(t) c^{\prime}(a(t)-c(a(t))) .\right.
\end{gathered}
$$

There are implicit constraints on the state variables $V$ and $W$, which both must be non-negative. There is a similar non-negativity constraint on the principal's control variable: the agent's effort level $a(t)$. We will not incorporate these constraints explicitly in the problem, but will ensure that they are satisfied when characterizing the optimal solution.

By Pontryagin's maximum principle, there must exist continuous functions (the co-state multipliers) attached to the first and the second constraint respectively, denoted by $\lambda(t)$ and $\mu(t)$, so that when the Hamiltonian

$$
\begin{aligned}
\mathcal{H}(t) \equiv \lambda(t)\left(\left(r_{P}+a(t)\right) V(t)-a(t)\right. & \left.\left(v-W(t)-c^{\prime}(a(t))\right)\right) \\
& +\mu(t)\left(r_{A} W(t)-\left(a(t) c^{\prime}(a(t))-c(a(t))\right)\right)
\end{aligned}
$$

is defined, the necessary conditions for optimality are given by:

$$
\begin{aligned}
\mu(0) & =0 ; \\
\frac{\partial \mathcal{H}}{\partial a} & =-\lambda\left(v-W-V-c^{\prime}(a)-a c^{\prime \prime}(a)\right)-\mu a c^{\prime \prime}(a)=0 ; \\
\dot{\lambda} & =\left(r_{P}+a\right) \lambda-\frac{\partial \mathcal{H}}{\partial V}=0 ; \\
\dot{\mu} & =\left(r_{P}+a\right) \mu-\frac{\partial \mathcal{H}}{\partial W}=\left(r_{P}-r_{A}+a\right) \mu-a \lambda .
\end{aligned}
$$

In these conditions, we have suppressed the time indices for notational convenience. We have also expressed the problem in current values terms, as the last two conditions (14) and (15) make clear. Also, in those conditions, the principal's effective discount rate is $r_{P}+a$, since in any interval $[t, t+d t)$, there is a probability $a(t) d t$ that the agent completes the project and the problem stops.

There is also a transversality condition; the form of this condition depends on whether it is optimal for the principal to stop the problem (i.e., fire the agent) in finite time; or whether an infinite horizon is optimal. Our first result in this Section considers this question, showing that it is not optimal to choose a finite stopping time.

\footnotetext{
${ }^{8}$ An alternative approach is to use the method developed by Spear and Srivastava (1987) and Phelan and Townsend (1991) and write the optimal contract in terms of the agent's continuation value as the state variable. In earlier versions of this paper, we used this second approach. Both approaches will give the same answer, but it turns out that using the optimal control approach makes comparison to sequentially rational equilibria (see Section 5) easier.
} 
Proposition 3 A finite stopping time is not optimal in the full commitment problem when $r_{P} \leq r_{A}$.

Proof. See the Appendix.

Proposition 3 shows that sufficient incentives can be provided to the agent by reducing his wage over time, without resorting to firing him. The result will allow us to establish unambiguously the dynamics of the optimal contract. The economic reasoning behind the result is as follows. Suppose that there is a time, $T$ say, when the agent's wage and hence effort falls to zero. Now suppose that the principal raises the wage at $T$ by a very small amount $d w>0$. To fix ideas, suppose that the cost function is quadratic, taking the form $c(a)=a^{2} / 2$. The increase in wage at time $T$ has a first-order effect on the agent's effort at $T$. Since the wage after $T$ is zero, the agent chooses effort $a(T)=d w$. Hence $W(T)=0.5(d w)^{2} \Delta$. But of course, the principal has to be concerned about the incentive effect on the agent at times before $T$. This effect is second-order, however. The agent's Bellman equation an instant before $T$ is

$$
W(T-d t)=\max _{a}\left\{a w(T-\Delta) \Delta-0.5 a^{2} \Delta+(1-a \Delta)\left(1-r_{A} \Delta\right)(d w)^{2} \Delta\right\} .
$$

Hence the agent's optimal choice of action at $T-\Delta$ is given by $w(T-\Delta)-$ $a-(d w)^{2}=0$, and so the incentive effect of a positive wage at $T$ can be made arbitrarily small. ${ }^{9}$

So, we know that the principal will never fire the agent. We shall now show that the dynamics of the full commitment solution are such that once the agent's value starts to increase, it must subsequently always increase. (That is, if $\dot{W}(t)>0$ at some $t$, then $\dot{W}\left(t^{\prime}\right)>0$ for all $t^{\prime}>t$.) But this is inconsistent with the transversality condition when the horizon is infinite, which essentially requires the optimal path to be bounded. This implies that the agent's value must be decreasing along the optimal path. The result that we find is (as we discuss in the introduction) similar to existing results from e.g., the optimal unemployment insurance literature. Nevertheless, we shall spend some time deriving the dynamics, for two reasons. First, and less importantly, our model is somewhat different from those in that literature, and so we have to establish the result for our setting. Secondly, and more importantly, a detailed understanding of the dynamics will prove very useful in the next Section, where we derive more novel results relating to sequentially rational equilibria.

To show this, we first state the appropriate transversality condition in this case. If $V^{*}(t)$ and $W^{*}(t)$ are optimal trajectories of the state variables, then

$$
\lim _{t \rightarrow \infty} \lambda(t)\left(V^{*}(t)-V(t)\right) \leq 0, \quad \lim _{t \rightarrow \infty} \mu(t)\left(W^{*}(t)-W(t)\right) \leq 0
$$

\footnotetext{
${ }^{9}$ This intuition relies on the feature that $c^{\prime}(0)=0$, while the result does not require this. Note that we have $v>c^{\prime}(0)$ in all interesting cases. To carry the intuition over, therefore, just interpret $v$ as being net of $c^{\prime}(0)$. We thank a referee for pointing out this issue to us.
} 
for all feasible trajectories $V(t)$ and $W(t)$. (See Kamihigashi (2001).) Less formally, the principal cannot gain by deviating from the optimal path given by $V^{*}(t)$ and $W^{*}(t)$ and never returning to it. This is clearly a necessary condition for optimality. Equally clearly, the optimal states must be bounded: the values to the principal and the agent cannot grow indefinitely, nor can they become negative. The implication for the problem at hand is that the system must converge to a steady state in which the controls and states are stationary and bounded.

We already have expressions for the dynamics of $V$ and $W$, as well as the costate variables. We now derive the differential equation for the optimal control $a$. To simplify the derivation, we set $\lambda$ equal to -1 ; this is without loss of generality. ${ }^{10}$ Then, differentiate the first-order condition (13) with respect to time:

$$
\left(c^{\prime \prime}(a)+(\mu+1)\left(c^{\prime \prime}(a)+a c^{\prime \prime \prime}(a)\right)\right) \dot{a}=-\dot{V}-\dot{W}-a c^{\prime \prime}(a) \dot{\mu} .
$$

We establish in the proof of Proposition 4 (see the appendix) that the Hamiltonian $\mathcal{H}$ is equal to zero along the optimal path. This means that we can write $\dot{V}$ as $\mu \dot{W}$. Doing this, and using equations (9) and (15) to substitute in for $\dot{W}(t)$ and $\dot{\mu}(t)$, gives the following differential equation for the dynamics of $a(t)$ :

$$
\begin{aligned}
& \left(c^{\prime \prime}(a)+(\mu+1)\left(c^{\prime \prime}(a)+a c^{\prime \prime \prime}(a)\right)\right) \dot{a} \\
& \quad=-(\mu+1)\left(r_{A} W-\left(a c^{\prime}(a)-c(a)\right)+a^{2} c^{\prime \prime}(a)\right)-a^{2} c^{\prime \prime}(a)\left(r_{P}-r_{A}\right) \mu .
\end{aligned}
$$

We are now able to characterise the dynamics of the optimal commitment contract when $r_{A} \geq r_{P}$. To do so, define the myopic effort level $a^{M}$ by $v-$ $\left.c^{\prime}\left(a^{M}\right)-a^{M} c^{\prime \prime}\left(a^{M}\right)=0\right)$. This is the effort level that would occur if both the principal and the agent ignored any continuation of the game beyond the current period. Then the marginal benefit from effort is $v$; the static marginal cost is $c^{\prime}(a)$; to this static marginal cost is added an agency cost, captured by $a c^{\prime \prime}(a)$. Hence the myopic effort level is less than the static effort $a^{S}$ that would occur in the single-agent problem, defined by $v=c^{\prime}\left(a^{S}\right)$.

Proposition 4 In the full commitment solution when $r_{A} \geq r_{P}$, the agent's continuation value $W(t)$, the optimal wage profile $w(t)$, and the agent's effort level $a(t)$ are all decreasing over time. If $r_{P}=r_{A}$, then the continuation value, wage and effort levels converge to zero. If $r_{P}<r_{A}$, then the wage and effort levels converge to strictly positive levels. The initial effort level, and hence all levels, are below the myopic effort $a^{M}$.

\footnotetext{
${ }^{10} \lambda$ must be a constant, from equation (14)). Clearly, then, the value of $\lambda$ can be set equal to any constant; the levels of other variables in the problem are then defined relative to this arbitrary choice of the value of $\lambda$. We choose $\lambda=-1$ as this turns out to be analytically convenient.
} 


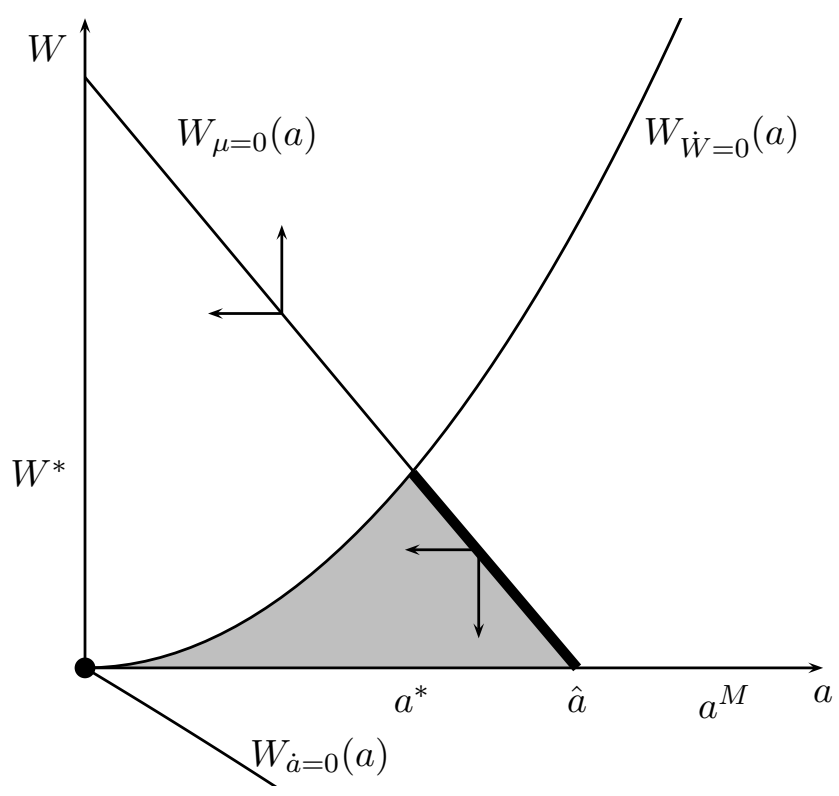

Figure 2: Phase diagram for the problem with full commitment with $r_{P}=r_{A}$; $v=g=1 ; r_{P}=r_{A}=0.05$

Proof. See the Appendix.

Figures 2 and 3 illustrate the proof of the Proposition. The phase diagrams involve two curves. The first, denoted $W_{\mu=0}=0(a)$, are the pairs of $(a, W)$ along which the multiplier $\mu$ attached to the state variable $W$ is zero. (See equation A.24 in the Appendix.) Since the initial value $W(0)$ is freely chosen, $\mu(0)=0$, and the optimal path must start somewhere on this curve. The second curve, denoted $W_{\dot{W}=0}=0(a)$, are the pairs of $(a, W)$ along which the state variable $W$ is stationary. (See equation (9).) Below this curve, $W$ is decreasing; above it, it is increasing. Since proposition 4 tells us that $W(t)$ is decreasing over time, we know that the optimal path must lie entirely below the $W_{\dot{W}=0}=0(a)$ curve.

In fact, in the shaded area in the figures, both $\dot{a}$ and $\dot{W}$ are non-positive. Moreover, the optimal initial point must lie on the portion of the $W_{\mu=0}=0(a)$ curve that is to the south-east of the $W_{\dot{W}=0}=0(a)$ curve. Any optimal path from that portion of the curve must move into the shaded area; hence $\dot{a}$ and $\dot{W}$ must be non-positive along the entire path. Possible steady states are marked with a dot; clearly, they must lie on the curve along which $\dot{W}=0$. If $r_{P}=r_{A}$, then only one steady state exists: the origin, with $a=W=0$. Otherwise, two steady states exist (as shown in the figure). The optimal path converges to the steady state with $a=W=0$, if $r_{P} \geq r_{A}$. Otherwise, it converges to a steady state with strictly positive levels of $a$ and $W$.

These results are reminiscent of those in the literature on optimal unemploy- 


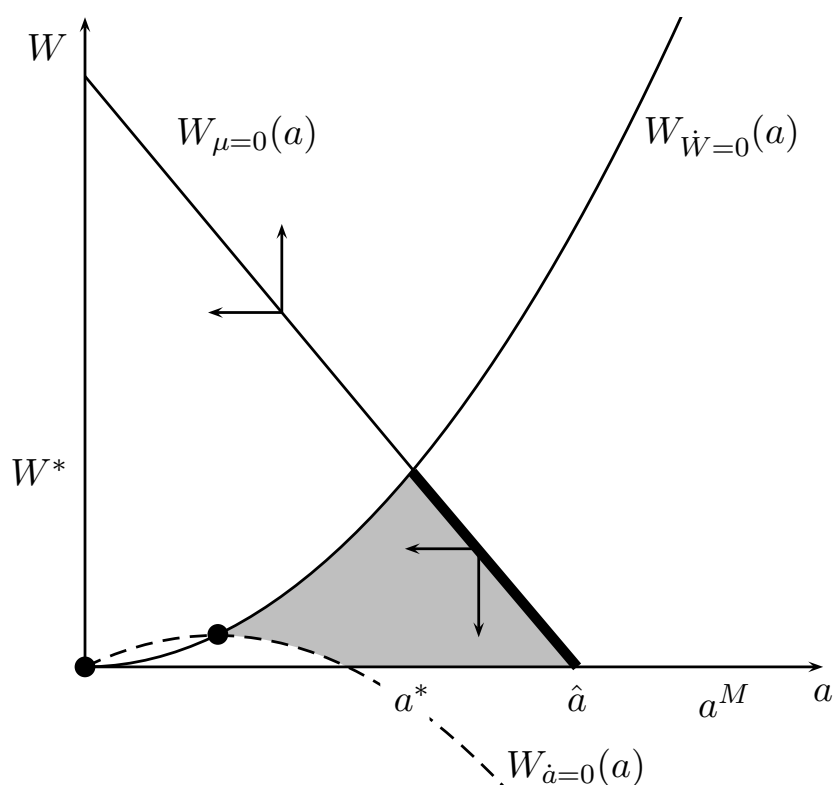

Figure 3: Phase diagram for the problem with full commitment with $r_{P}<r_{A}$; $v=g=1 ; r_{P}=0.02, r_{A}=0.05$

ment literature (reviewed in the introduction). In particular, the idea that the principal uses a declining wage to incentivize the agent can be found in e.g., Shavell and Weiss (1979). The mechanics are somewhat different here, however; and the analysis here is somewhat more general, involving different discount rates. Because the agent has increasing marginal cost of effort, he looks to smooth his effort over time: to substitute away from current effort toward future effort. Limited liability means that the agent earns positive rents from the contract. The principal uses the dynamics of these rents to provide the agent with incentives to exert effort. In particular, the full commitment contract ensures that the agent's continuation value is decreasing in equilibrium. By these means, the principal gives the agent incentives to exert current effort. The continuation value is driven downwards by a decreasing wage; the agent's effort also decreases over time.

The profile of effort should be contrasted to the efficient solution analysed in the previous Section. There, we showed that the efficient effort either is constant (when $r_{P}=r_{A}$ ) or grows exponentially (when $r_{P}<r_{A}$ ).

The Proposition distinguishes between two cases: when the principal and agent are equally patient $\left(r_{P}=r_{A}\right)$, and when the agent is more impatient. In the former case, in the full commitment solution, the agent's wage and action go to zero in the long run. In the latter case, the wage and action converge towards strictly positive levels. The intuition for this difference lies in how the principal can reward the agent for effort in a way that matches their relative time 


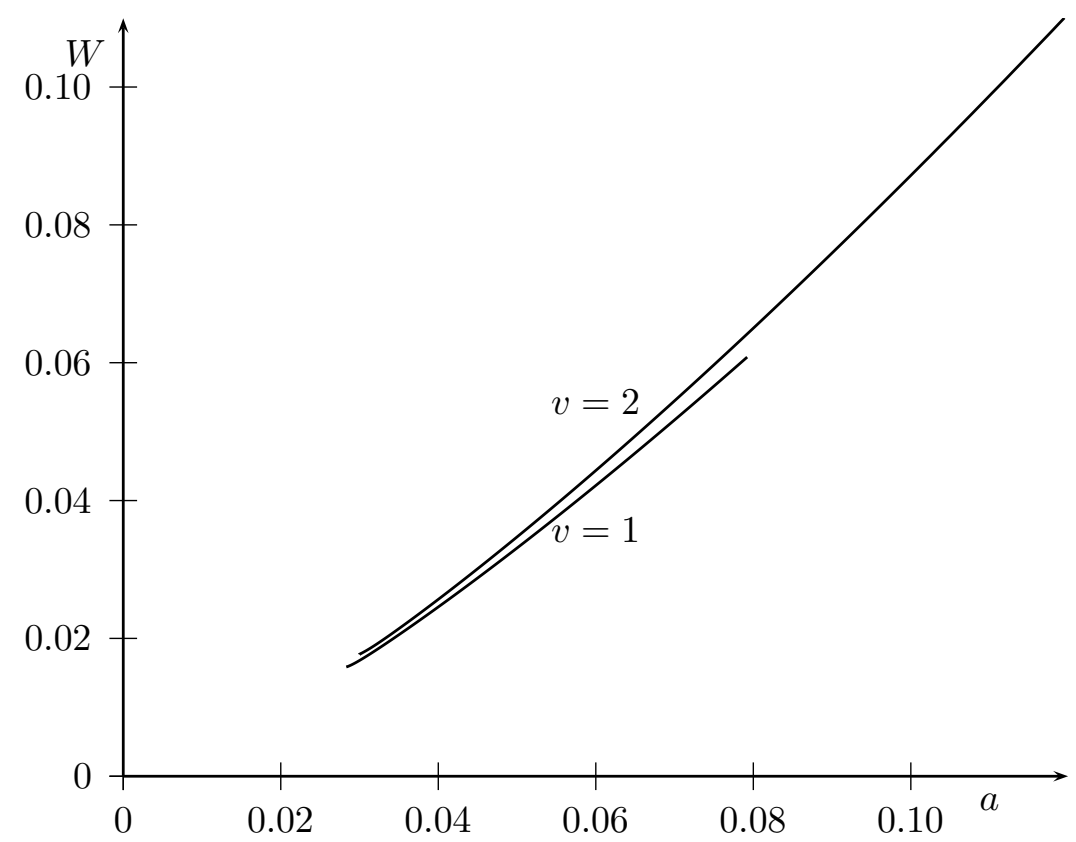

Figure 4: Numerical solutions for the full commitment case; $g=1 ; r_{P}=$ $0,02, r_{A}=0.05$

preferences. When the agent is less patient than the principal $\left(r_{A}>r_{P}\right)$, the principal can allow the agent a positive value in the long-run, without affecting (too much) incentives towards current effort. And by allowing the long-run value to be positive, the principal ensures that the agent's effort is always bounded away from zero. For the more patient principal, the latter is important.

Figure 4 shows numerical solutions for the full commitment problem for two different values of the model parameter $v{ }^{11}$ When $v=1$, for example, the initial effort $a(0)$ exerted by the agent is 0.07956 . The equilibrium effort declines monotonically over time, until it reaches its steady state value of 0.02832 . The agent's equilibrium value declines monotonically from $W(0)=0.06115$ to its steady state value of 0.01586 . The principal's value (not shown in the figure) declines from $V(0)=0.6195$ to its steady state value of 0.5324 . When $v=2$, values and actions are higher (which makes sense), but show the same dynamics. Figure 5 shows the equilibrium values over time, figure 6 the equilibrium action and wage over time.

The final result in this Section examines whether the agent completes the project in finite time.

\footnotetext{
${ }^{11}$ We have assumed a quadratic cost function: $c(a)=\gamma a^{2}$, where $\gamma$ is set at 1 . The discount rates in this figure are $r_{A}=5 \%, r_{P}=2 \%$. Details of the numerical work, including the Matlab code, are available from the authors on request.
} 


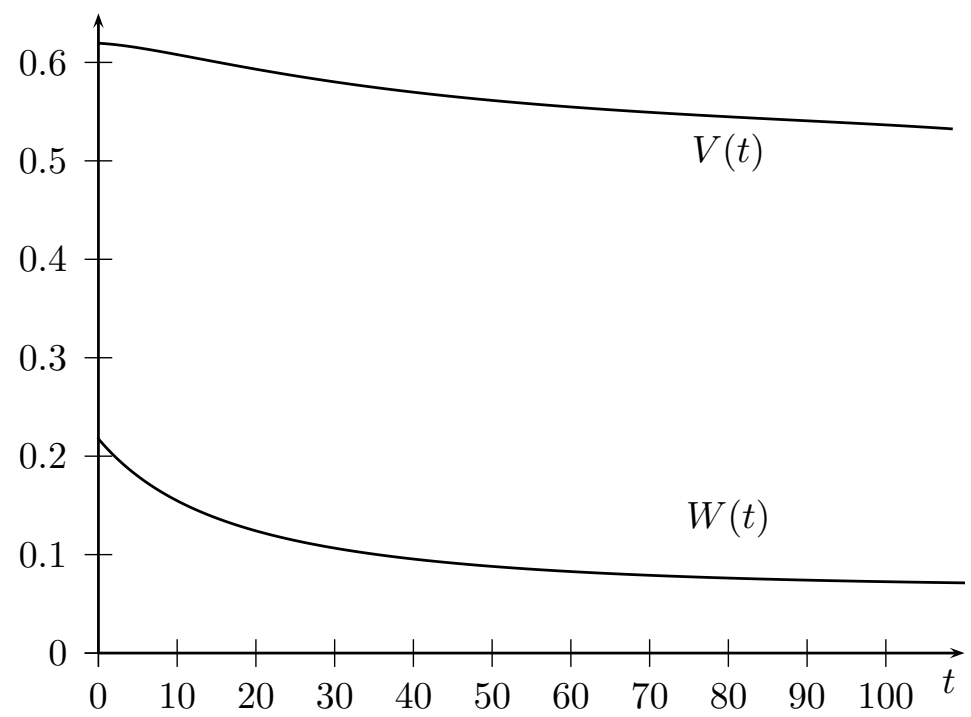

Figure 5: Numerical solutions for values, in the full commitment case; $v=g=$ $1 ; r_{P}=0,02, r_{A}=0.05$

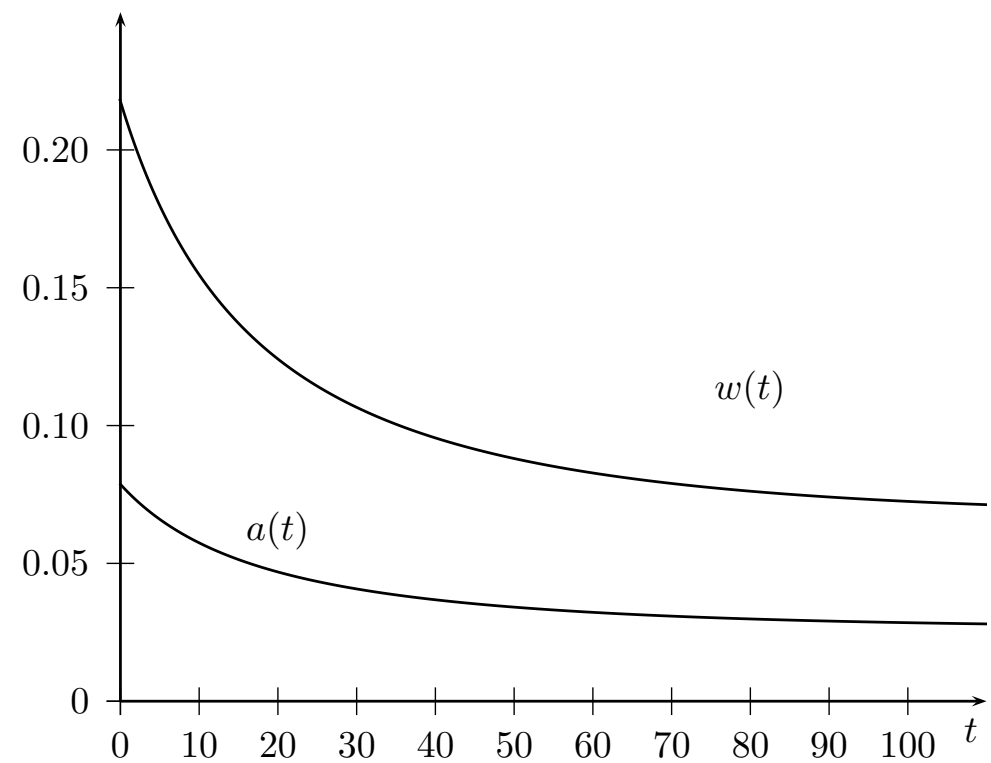

Figure 6: Numerical solutions for the action and wage, in the full commitment case; $v=g=1 ; r_{P}=0,02, r_{A}=0.05$ 
Proposition 5 When the principal faces an impatient agent $\left(r_{P}<r_{A}\right)$, the project is completed in finite time almost surely. When the principal faces an equally patient agent $\left(r_{P}=r_{A}\right)$, if the cost function is not too convex (i.e., if $c(a)=O\left(a^{4-\beta}\right)$ for some $\left.\beta<2\right)$, then the probability that the project is completed in finite time is less than one. Otherwise, if $\beta \geq 2$, then the project is completed in finite time almost surely.

Proof. See the Appendix.

The Proposition follows immediately when the agent is relatively impatient, since his effort is always strictly positive. The proof is more subtle when the agent is relatively patient, since in this case, his effort declines to zero. The proof relies on looking at the rate of decline of the agent's effort as the steady state is approached. The intuition is that if costs are highly convex, it is costly for the principal to induce the agent to have big differences in his effort across time. As a consequence, the agent's effort decreases relatively slowly in equilibrium (at least near the steady state). This means that $\int_{o}^{\infty} a(t) d t$ is unbounded and so the project completes almost surely. But when costs are not too convex, it is not too costly for the principal to induce high current effort and lower future effort. This means that the agent's effort decreases relatively quickly in equilibrium (near the steady state); consequently, $\int_{o}^{\infty} a(t) d t$ is bounded and the probability that the project completes is less than 1 . As we noted in the introduction, certain projects (e.g., the nuclear power plant in Olkiluoto, Finland) can experience significant delay; time will tell if this particular project is ever completed.

\section{$5 \quad$ Wages without commitment}

In the previous Section, we assumed that the principal can commit to an entire path of future wages. While full commitment is the standard assumption in static mechanism design problems, relaxing this assumption is quite natural in dynamic settings. When the agent is as patient as the principal, we have seen that the optimal commitment wage decreases towards zero. This implies that the optimal effort exerted by the agent and the continuation value to the principal also converge to zero. In these circumstances, the principal would be tempted to offer a temporary bonus to the agent for completing the task.

In this Section, we make the opposite extreme assumption on commitment: the principal can promise only temporary spot wages in each period that condition on whether the project was successfully completed. (More precisely, we require that all continuation wage offer strategies, on and off the equilibrium path, are best responses to the agent's strategy.) In order to analyze the principalagent problem in this case, we must make use of the full machinery of repeated extensive-form games to pin down the principal's and the agent's behaviour after all possible wage offers. 
Even though the interaction between the principal and the agent induces a stochastic game between the parties, the principal has still the power to set incentives for the agent through the wage offers. In the absence of commitment, the game has a non-degenerate set of equilibria. In this Section, we characterise the set of all sequentially rational equilibria through an optimal penal code resembling the construction in Abreu (1988). We also compute the best sequentially rational equilibrium from the principal's point of view. This choice reflects the common convention from the literature on mechanism design, that the designer also proposes the equilibrium to be played. Later in this Section, we consider renegotiation between the principal and the agent.

A sequentially rational equilibrium in our game is a pair $(\mathbf{w}, \mathbf{a})$ such that $\left.\mathbf{w}\right|_{h^{t}}$ is optimal given $\left.\mathbf{a}\right|_{\left(h^{t}, w_{t}\right)}$ for all ${ }_{\left(h^{t}, w_{t}\right)}$, and $\left.\mathbf{a}\right|_{\left(h^{t}, w_{t}\right)}$ is optimal given $\left.\mathbf{w}\right|_{h^{t}}$ and $w_{t}$ for all $\left(h^{t}, w_{t}\right)$. Since the underlying contracting game is stationary, in the sense that the continuation game after each history (conditional on no success) is strategically equivalent to the original game, we may view the set of equilibrium continuation wage setting strategies in a recursive manner. The set of sequentially rational equilibrium strategies does not depend on $h^{t}$. We denote the set of equilibrium strategies by $\mathcal{E}$. Then every equilibrium continuation strategy $(\mathbf{w}, \mathbf{a}) \in \mathcal{E}$ after history $h^{t}$ can be written as ${ }^{12}$

$$
\left(\left.\mathbf{w}\right|_{h^{t}},\left.\mathbf{a}\right|_{\left(h^{t}, w_{t}\right)}\right)=\left(\left(\mathbf{w}^{s}\left(h^{t}\right), \mathbf{w}^{f}\left(h^{t}\right), \mathbf{a}\left(h^{t}, w_{t}^{s}, w_{t}^{f}\right)\right),(\widehat{\mathbf{w}}, \widehat{\mathbf{a}})\right) \text { for some }(\widehat{\mathbf{w}}, \widehat{\mathbf{a}}) \in \mathcal{E} .
$$

Our task is to characterize pairs $(V(\mathbf{w}, \mathbf{a}), W(\mathbf{w}, \mathbf{a}))$ of payoffs induced by some $(\mathbf{w}, \mathbf{a}) \in \mathcal{E}$.

\subsection{The worst sequentially rational equilibrium for the principal}

In order to identify the worst equilibrium for the principal, we start by showing that the game has a unique stationary sequentially rational equilibrium, in which the principal offers the same wage contracts in all periods and the agent conditions his current effort choice on the current wage offers and the stationary wage strategy for the future periods. (In notation, a stationary equilibrium is such that $\mathbf{w}^{f}\left(h^{t}\right), \mathbf{w}^{s}\left(h^{t}\right)$ and $\mathbf{a}\left(h^{t}\right)$ are independent of $h^{t}$.) We then show that this stationary equilibrium yields the lowest payoff to the principal of all sequentially rational equilibria.

Proposition 6 The game has a unique stationary equilibrium where $\mathbf{w}^{s}\left(h^{t}\right)=$ $w^{S R}>0$, and $\mathbf{w}^{f}\left(h^{t}\right)=0$ for all $h^{t}$ and histories and $\mathbf{a}\left(h^{t}, w_{t}\right)=a^{S R}\left(h^{t}, w_{t}\right)>0$ after all histories $h^{t}$ where $\mathbf{w}^{s}\left(h^{t}\right)=w^{S R}>0$ and $\mathbf{w}^{f}\left(h^{t}\right)=0$.

Proof. See the Appendix.

\footnotetext{
${ }^{12}$ Since our stage game is in extensive form, sequential rationality also requires that for any $w_{t}$, the continuation $(\widehat{\mathbf{w}}, \mathbf{a})$ constitutes an equilibrium in the game.
} 
Denote the payoffs in this equilibrium by $V^{S R}$ and $W^{S R}$. Our second result shows that the principal's payoff in the (unique) stationary equilibrium is the lowest payoff of any equilibrium in the set of sequentially rational equilibria. In the course of proving this result, we show that all wage paths that generate higher payoffs to the principal than the stationary equilibrium can be supported as sequentially rational equilibria. This turns out to be quite useful for characterizing the best sequentially rational equilibrium for the principal. In particular, we will be able to use the laws of motion (equations (9) and (10)) established in the previous Section to describe the sequentially rational equilibrium path with the highest payoff to the principal.

Proposition 7 All sequentially rational equilibria induce a payoff of at least $V^{S R}$ to the principal.

Proof. See the Appendix.

It should be noted that even though the worst equilibrium payoff to the principal can be supported as a stationary equilibrium, the same is not true of the best payoff. In the proof of Proposition 7, a key step establishes that a relatively high equilibrium continuation payoff to the principal can be reduced by an essentially unconditional monetary transfer from the principal to the agent. To get a similar mechanism to work for the best equilibria from the principal's point of view, we would need a mechanism that transfers continuation value unconditionally from the agent to the principal. This is, however, ruled out by our assumption of limited liability.

\subsection{The best sequentially rational equilibrium for the principal}

The problem of characterizing the best sequentially rational equilibrium for the principal is conceptually similar to the full commitment problem in the previous Section. By Lemma 3 in the Appendix, the principal can implement all wage and effort paths that satisfy the requirement that $V(t) \geq V^{S R}$ for all $t$. The strategies supporting these equilibria take a simple form. As long as the principal has offered wages on the equilibrium path, both players continue to choose actions on the path. If the principal deviates at any point, then play reverts to the stationary equilibrium. Since this is the worst equilibrium for the principal, it ensures that no deviation takes place.

Note also that, in common with the full commitment solution, no payment is made at any point on the equilibrium path of the best sequentially rational equilibrium at which the project has not been completed. ${ }^{13}$

\footnotetext{
${ }^{13}$ The argument is very similar: suppose to the contrary that $w^{f}\left(h^{t}\right)>0$ for some history $h^{t}$ on equilibrium path. Then, using a previous argument, reduce $w^{s}\left(h^{t}\right)$ and $w^{f}\left(h^{t}\right)$ by the same amount, leaving all other wages constant. This has the effect of increasing effort in earlier stages while reducing the principal's expected payment. To complete the argument for the sequentially rational case, note that the punishment path following any deviation by the principal is the
} 
It is easy, therefore, to express the maximization problem of the principal in a manner analogous to the previous Section. Consider then the following constrained optimization problem:

$$
\begin{aligned}
& \mathcal{S R}: \max _{\{a(t), W(0)\}} V(0) \quad \text { subject to } \\
& \dot{V}(t)=\left(r_{P}+a(t)\right) V(t)-a(t)\left(v-W(t)-c^{\prime}(a(t))\right), \\
& \dot{W}(t)=r_{A} W(t)-\left(a(t) c^{\prime}(a(t)-c(a(t))) .\right. \\
& V(t) \geq V^{S R}, \text { for all } t .
\end{aligned}
$$

If the last inequality constraint is not binding, then the problem is identical to the one solved in previously. Since the constraint binds, there is a finite $T$ such that $V(t)=V^{S R}$ for all $t \geq T$. The following Lemma determines the agent's continuation payoff at $T$. In the Lemma, and the rest of the section, we let $\underline{W}$ be the lowest payoff to the agent in any sequentially rational equilibrium. (Clearly, $\underline{W}$ exists and is well-defined.)

Lemma 2 The best sequentially rational equilibrium payoff to the principal is obtained by setting $W(T)=\underline{W}$.

Proof. See the Appendix.

Hence we have argued that the solution to $\mathcal{S R}$ satisfies the state constraints $(V(T), W(T))=\left(V^{S R}, \underline{W}\right)$. The state evolution equations in $\mathcal{S R}$ determine the values $V(t)$ and $W(t)$ for any given path of actions $\{a(t)\}$. Hence the problem of finding the best sequentially rational equilibrium, from the principal's point of view, can be written as one of finding the optimal sequence of actions and an optimal time $T$ when the constraint is hit; that is,

$$
\begin{aligned}
& \mathcal{S R}: \max _{\{a(t), T\}} V(0) \quad \text { subject to } \\
& \dot{V}(t)=\left(r_{P}+a(t)\right) V(t)-a(t)\left(v-W(t)-c^{\prime}(a(t))\right), \\
& \dot{W}(t)=r_{A} W(t)-\left(a(t) c^{\prime}(a(t)-c(a(t))) .\right. \\
& V(T)=V^{S R}, W(T)=\underline{W} .
\end{aligned}
$$

The implied laws of motion up to time $T$ in this best sequentially rational equilibrium satisfy the differential equations derived for the full commitment solution in the previous Section. Hence the qualitative properties of the wage and action sequences are similar. We summarize the findings in this subsection in the following Proposition:

stationary equilibrium, in which $w^{f}=0$ following all histories: see Proposition 6 . Hence we can conclude that $w^{f}\left(h^{t}\right)=0$ in the best sequentially rational equilibrium for all $h^{t}$, on (and off) the equilibrium path. 
Proposition 8 The action path with the highest expected payoff to the principal over all sequentially rational equilibria is given by the solution to $\mathcal{S R}$. Hence the equilibrium wage, action and values all decrease before time T. From T onwards, the continuation payoffs are given by $\left(V^{S R}, \underline{W}\right)$.

\subsection{Renegotiation-proof equilibria}

We now consider equilibrium when renegotiation of the initial contract is possible. Since there are different definitions for renegotiation-proofness in infinitehorizon games, we must select a concept to use. The weakest notion of renegotiationproofness is defined in Farrell and Maskin (1989). Since the wage offer $w$ currently on table is a part of the payoff relevant description of the continuation game, we must consider different histories up to $w$ when computing the agent's continuation strategy. An equilibrium $(\mathbf{w}, \mathbf{a}) \in \mathcal{E}$ is weakly renegotiation-proof (WRP) if for all $(h, w),\left(h^{\prime}, w\right) \in H$,

$$
\begin{array}{cc}
V\left(\left.\mathbf{w}\right|_{h},\left.\mathbf{a}\right|_{(h, w)}\right)>V\left(\left.\mathbf{w}\right|_{h^{\prime}},\left.\mathbf{a}\right|_{\left(h^{\prime}, w\right)}\right) & \Rightarrow W\left(\left.\mathbf{w}\right|_{h},\left.\mathbf{a}\right|_{(h, w)}\right)<W\left(\left.\mathbf{w}\right|_{h^{\prime}},\left.\mathbf{a}\right|_{\left(h^{\prime}, w\right)}\right), \\
W\left(\left.\mathbf{w}\right|_{h},\left.\mathbf{a}\right|_{(h, w)}\right)>W\left(\left.\mathbf{w}\right|_{h^{\prime}},\left.\mathbf{a}\right|_{\left(h^{\prime}, w\right)}\right) & \Rightarrow V\left(\left.\mathbf{w}\right|_{h},\left.\mathbf{a}\right|_{(h, w)}\right)<V\left(\left.\mathbf{w}\right|_{h^{\prime}},\left.\mathbf{a}\right|_{\left(h^{\prime}, w\right)}\right) .
\end{array}
$$

Hence a sequentially rational equilibrium is weakly renegotiation-proof if no pair of continuation payoffs (on or off the equilibrium path) is Pareto-dominated by another pair of continuation payoffs.

Since the equilibrium payoffs of Proposition 8 to both the principal and the agent are decreasing over time, this equilibrium is not renegotiation-proof. Nevertheless, it is clear from the definition of weak renegotiation-proofness and the characterization of sequentially rational equilibria in Lemma 3 in the Appendix that there are many WRP equilibria. For example, the following construction yields a WRP equilibrium with constant wage offers on the equilibrium path: punish any deviation by the principal by switching to the continuation game in which the unique stationary equilibrium of the game is played. Then any constant wage offer $w\left(h^{t}\right)=w$ on the equilibrium path, with the agent choosing the (stationary) action $a^{B R}(w)$ (where this notation denotes the agent's best response to the current and future constant wage offers $w)$, together form a WRP equilibrium of the game if $V(w, a(w))>V^{S R}$ and $W\left(w, a^{B R}(w)\right)<W^{S R}$.

We close this Section by discussing the best WRP equilibrium from the principal's point of view. Since all continuation equilibria of a WRP equilibrium are also WRP, it must be the case that in the best WRP equilibrium,

$$
V(0) \geq V(t) \text { for all } t \geq 0 \text {. }
$$

In the Appendix, we show that in fact $V(t)=V(0)$ and $W(t)=W(0)$ for all $t$ in the WRP with the maximal payoff to the principal. Consider the problem where the principal maximizes over constant wage sequences, taking into account that the agent best-responds. In other words, let

$$
w^{W R P} \equiv \arg \max _{w} V\left(w, a^{B R}(w)\right) .
$$


Proposition 9 The wage path with the highest expected payoff to the principal over all weakly renegotiation-proof equilibria is given by the pair of strategies:

$$
(w(t), a(w, t))= \begin{cases}\left(w^{W R P}, a^{B R}\left(w^{W R P}\right)\right) & \text { if } w\left(t^{\prime}\right)=w^{W R P} \text { for all } t^{\prime} \leq t \\ \left(w^{S R}, a\left(w, W^{S R}\right)\right) & \text { otherwise. }\end{cases}
$$

Proof. See the Appendix.

There are two parts to the argument. First, as we have already noted, it must be that $V(0) \geq V(t)$ for all $t$ in the best WRP equilibrium (since all continuation equilibria of a WRP equilibrium are also WRP). Secondly, the proof shows that there cannot be a time $t$ such that $V(t)<V(0)$ : if there were, then a new continuation game could be constructed, starting at the last time $\tau$ where $V(\tau)=V(0)$, with a continuation value of $V(\tau)$ for the principal. But then the initial payoff to the principal in this equilibrium would be greater than $V(0)$ : a contradiction. Hence we find the typical property of renegotiation proof contracts: payments on the equilibrium path must be constant. See Shapiro and Stiglitz (1984) and MacLeod and Malcomson (1998) for similar results when contracts can be "restarted".

\section{Conclusions}

We have developed a model of dynamic moral hazard involving project completion which has allowed us to identify clearly the intertemporal incentives involved. We show in the full commitment solution how the principal controls the agent's continuation value over time to provide the optimal dynamic incentives for current effort. The long-run outcome of these dynamics is determined by the relative discount rates of the principal and the agent. The principal never fires the agent - despite the fact that, when the agent is relatively patient, there may be a positive probability that the project is never completed. We also characterise the set of sequentially rational equilibria, in particular identifying the minimal and maximal payoffs of the principal, including when renegotiation is possible.

The framework that we have developed is very tractable and offers a base from which we intend to explore further dynamic incentives for this type of problem. 


\section{Appendix: Proofs}

\section{A formal derivation of the continuous-time limit for the full com- mitment solution}

Following Biais et al. (2007), we prove the convergence result in the dynamic programming formulation with the agents' promised utility as the state variable. Consider the principal's dynamic programming problem in the discretetime model with period length $\Delta$ :

$$
V^{\Delta}(W)=\max _{a^{\Delta}, w^{\Delta}, W_{-}^{\Delta}}\left\{\Delta a^{\Delta}\left(v-w^{\Delta}\right)+\left(1-a^{\Delta} \Delta\right)\left(1-r_{P} \Delta\right) V^{\Delta}\left(W_{-}^{\Delta}\right)\right\}:=T^{\Delta} V^{\Delta}
$$

subject to

$$
\begin{aligned}
c^{\prime}\left(a^{\Delta}\right) & \geq w^{\Delta}-W_{-}^{\Delta}, c^{\prime}\left(a^{\Delta}\right)=w^{\Delta}-W_{-}^{\Delta} \text { if } a^{\Delta}>0, \\
W & =\Delta a^{\Delta} w^{\Delta}-c\left(a^{\Delta}\right) \Delta+\left(1-\Delta a^{\Delta}\right)\left(1-r_{A} \Delta\right) W_{-}^{\Delta}, \\
w^{\Delta}, W_{-}^{\Delta} & \geq 0 .
\end{aligned}
$$

The notation here expresses the dependence of variables on the period length $\Delta$; for example, $a^{\Delta}$ is the equilibrium choice of effort. $W_{-}^{\Delta}$ denotes the agent's value in the subsequent period.

Since each $V$ is bounded above by $v$ and bounded below by $W$, we see that $T^{\Delta}$ maps bounded functions into bounded functions. Since the constraint set is a continuous correspondence of $W$, we see by Berges maximum theorem that $T^{\Delta}$ maps continuous functions into continuous functions. By Blackwell's sufficient conditions, it is easy to check that $T^{\Delta}$ is a contraction and hence has a unique fixed point $V^{\Delta}$.

Consider next the following differential equation:

$$
r_{p} V(W)=\max _{a}\left\{a\left(v-c^{\prime}(a)-W-V(W)\right)+\left(r_{A} W-a c^{\prime}(a)+c(a)\right) V^{\prime}(W)\right\} .
$$

The first-order condition for the maximization problem is $\left(v-c^{\prime}(a)-W-\right.$ $V(W))-a c^{\prime \prime}(a)\left(V^{\prime}(W)+1\right)=0$, or

$$
c^{\prime}(a)+a c^{\prime \prime}(a)\left(V^{\prime}(W)+1\right)=(v-W-V(W)) .
$$

The problem is concave in $a$ since $\left(V^{\prime}(W)+1\right) \geq 0$.

Since the left-hand side of equation (A.23) is an unbounded increasing function in $a$ and the right-hand side is a positive, a solution $a\left(V(W), V^{\prime}(W), W\right)$ exists. Substituting into equation (A.22), we get a non-linear ordinary differential equation. The phase diagram analysis shows that for each $W_{0}$, we have a unique value $V_{0}=V\left(W_{0}\right)$ such that the solution does not violate transversality conditions. We let $V$ denote the unique feasible solution of the differential equation. 
Our main goal in this Section is to show that $V^{\Delta}$ converges uniformly to $V$ as $\Delta \rightarrow 0$. We start by analyzing $T^{\Delta} V$. From equation (A.21), we get $W-(1-$ $\left.r_{A} \Delta\right) W_{-}^{\Delta}=\Delta a^{\Delta} c^{\prime}\left(a^{\Delta}\right)-c\left(a^{\Delta}\right) \Delta+\Delta^{2} a^{\Delta} r_{A} W_{-}^{\Delta}$, or

$$
W_{-}^{\Delta}-W=\frac{r_{A} \Delta}{\left(1-r_{A} \Delta\right)} W-\frac{\Delta a^{\Delta} c^{\prime}\left(a^{\Delta}\right)-c\left(a^{\Delta}\right) \Delta+\Delta^{2} a^{\Delta} r_{A} W_{-}^{\Delta}}{\left(1-r_{A} \Delta\right)} .
$$

Since $a^{\Delta}$ is bounded, we can ignore second-order terms as $\Delta \rightarrow 0 .{ }^{14}$ Thus

$$
\lim _{\Delta \rightarrow 0} \frac{W_{-}^{\Delta}-W}{\Delta}=r_{A} W-a^{\Delta} c^{\prime}\left(a^{\Delta}\right)-c\left(a^{\Delta}\right) .
$$

Since $V$ is differentiable (by construction), we can write

$$
\begin{aligned}
& T^{\Delta} V(W)=\max _{a^{\Delta}, W_{-}^{\Delta}}\left\{\Delta a^{\Delta}\left(v-c^{\prime}\left(a^{\Delta}\right)-W-\left(r_{A} W-a^{\Delta} c^{\prime}\left(a^{\Delta}\right)-c\left(a^{\Delta}\right)\right) \Delta\right)\right. \\
& \left.\quad+\left(1-a^{\Delta} \Delta\right)\left(1-r_{P} \Delta\right)\left(V(W)+\left(r_{A} W-a^{\Delta} c^{\prime}\left(a^{\Delta}\right)-c\left(a^{\Delta}\right)\right) \Delta V^{\prime}(W)\right)\right\} .
\end{aligned}
$$

Ignoring second-order terms, we have

$$
\begin{aligned}
T^{\Delta} V(W)= & \max _{a^{\Delta}, w^{\Delta}, W_{-}^{\Delta}}\left\{\Delta a^{\Delta}\left(v-c^{\prime}\left(a^{\Delta}\right)-W-V(W)\right)\right. \\
& +V(W)-r_{P} \Delta V(W)+\left(r_{A} W-a^{\Delta} c^{\prime}\left(a^{\Delta}\right)-c\left(a^{\Delta}\right)\right) \Delta V^{\prime}(W) .
\end{aligned}
$$

Hence we have

$$
\begin{aligned}
\max _{W} \frac{\left|T^{\Delta} V(W)-V(W)\right|}{\Delta}= & \max _{W} \max _{a^{\Delta}}\left\{a^{\Delta}\left(v-c^{\prime}\left(a^{\Delta}\right)-W-V(W)\right)\right. \\
& -r_{P} V(W)+\left(r_{A} W-a^{\Delta} c^{\prime}\left(a^{\Delta}\right)-c\left(a^{\Delta}\right)\right) V^{\prime}(W) .
\end{aligned}
$$

But by the maximization problem in the continuous time differential equation (A.22), the right-hand side is zero. Hence $T^{\Delta} V$ converges to $V$ uniformly. Notice next that $d\left(V^{\Delta}, V\right) \leq d\left(V^{\Delta}, T^{\Delta} V\right)+d\left(T^{\Delta} V, V\right)$. Since $T^{\Delta} V^{\Delta}=V^{\Delta}$ and since $T^{\Delta}$ is a contraction with factor $\left(1-r_{P} \Delta\right)$, we have $d\left(V^{\Delta}, T^{\Delta} V\right) \leq$ $\left(1-r_{P} \Delta\right) d\left(V^{\Delta}, V\right)$. Putting these together gives $d\left(V^{\Delta}, V\right) \leq d\left(T^{\Delta} V, V\right) / r_{P} \Delta$, so we have

$$
\lim _{\Delta \rightarrow 0} d\left(V^{\Delta}, V\right) \leq \frac{1}{r_{P}} \lim _{\Delta \rightarrow 0} \frac{d\left(T^{\Delta} V, V\right)}{\Delta}=0 .
$$

This completes the proof.

\footnotetext{
${ }^{14}$ It is never optimal to induce the agent to choose an effort $a$ where $c^{\prime}(a)>v$, since it is always possible to give the agent the same expected payoff in the form of an unconditional transfer (i.e., one paid with or without success).
} 


\section{Proof of Proposition 3}

Suppose not i.e., suppose that it is optimal to choose a finite stopping time $T$. We show that this leads to a contradiction.

Since $W(T)$ can be freely chosen by the principal (subject only to a nonnegativity constraint), it must be that $\mu(T)=0$. Hence $\mu(0)=\mu(T)=0$ (recalling that $W(0)$ also is freely chosen.) Equation (15) tells us that $\dot{\mu}(0)=$ $-\lambda a(0)$ and $\dot{\mu}(T)=-\lambda a(T)$. Since $a(0)$ and $a(T)$ are non-negative and $\lambda \neq 0$, this means that either $\dot{\mu}(0)$ and $\dot{\mu}(T)$ are both positive or they are both negative (determined by the sign of $\lambda$ ). We analyse fully the former case; the latter case follows an identical argument. So, suppose $\dot{\mu}(0)$ and $\dot{\mu}(T)$ are both positive (and $\mu(0)=\mu(T)=0)$. Since $\mu$ is a continuous function of $t$, there must be some $t^{*} \in(0, T)$ such that $\mu\left(t^{*}\right)=0$, and $\mu(\cdot)$ is decreasing in $t$ at this point. But the latter cannot be the case, since equation (15) implies that $\dot{\mu}\left(t^{*}\right)=-\lambda a\left(t^{*}\right) \geq 0$.

Hence a contradiction has been established, and so a finite stopping time $T$ cannot be optimal.

\section{Proof of Proposition 4}

The proof uses a phase diagram in $(a, W)$ space. Three aspects need to be analysed: the dynamics of $a$ and $W$; and the sign of $V^{\prime}(\cdot)$ in equilibrium.

The sign of $\dot{W}$ is, from equation (9), determined by the sign of $r_{A} W-\left(a c^{\prime}(a)-\right.$ $c(a))$. This defines an upward-sloping function in $(a, W)$ space, given by

$$
W_{\dot{W}=0}(a) \equiv \frac{a c^{\prime}(a)-c(a)}{r_{A}}
$$

so that for $W>(<) W_{\dot{W}=0}(a), \dot{W}>(<) 0$. Note that $W_{\dot{W}=0}=0$.

We now determine the sign of $\mu$ in equilibrium. We first establish that the Hamiltonian $\mathcal{H}(t)$ is zero along the optimal path. Differentiation of $\mathcal{H}(t)$ gives $\dot{\mathcal{H}}(t)=-\ddot{V}(t)+\dot{\mu}(t) \dot{W}(t)+\mu(t) \ddot{W}(t)$. (Here, a double dot denotes a second derivative with respect to time.) After substitution of the expressions for the various terms and simplification, we end up with $\dot{\mathcal{H}}(t)=\left(r_{P}+a(t)\right) \mathcal{H}(t)$. There are then two possibilities: either $\mathcal{H}(t)>0$ or $\mathcal{H}(t)=0$ for all $t$. If the former, then $\mathcal{H}(t)$ grows at least exponentially over time; this clearly cannot be the case on any feasible path. Therefore $\mathcal{H}(t)=0$ for all $t$.

We can therefore write $\dot{V}$ as $\mu \dot{W}$. Now use equations (10) and (13) to give

$\left(r_{A} W-a c^{\prime}(a)+c(a)+\left(a+r_{P}\right) a c^{\prime \prime}(a)\right) \mu=r_{P}\left(v-W-c^{\prime}(a)\right)-\left(a+r_{P}\right) a c^{\prime \prime}(a)$.

Consider the left-hand side of this equation. From assumption $1, r_{A} W-a c^{\prime}(a)+$ $c(a)+\left(a+r_{P}\right) a c^{\prime \prime}(a) \geq 0$ for all non-negative values of $a$ and $W$. Hence the sign of $\mu$ is determined by the sign of $r_{P}\left(v-W-c^{\prime}(a)\right)-\left(a+r_{P}\right) a c^{\prime \prime}(a)$. This 
defines a function in $(a, W)$ space, given by

$$
W_{\mu=0}(a) \equiv v-c^{\prime}(a)-\left(\frac{a+r_{P}}{r_{P}}\right) a c^{\prime \prime}(a) .
$$

This is a downward-sloping function, with an intercept $W_{\mu=0}(0)=v$; it hits the horizontal axis at an effort level $\hat{a}$ strictly less than the myopic level $a^{M}$ (defined by $\left.v-c^{\prime}\left(a^{M}\right)-a^{M} c^{\prime \prime}\left(a^{M}\right)=0\right)$. For values of $(a, W)$ lying below (above) this function, $\mu$ is positive (negative); along the function, $\mu=0$. The function $W_{\dot{W}=0}(a)$ is, therefore, split into two portions by the function $W_{\mu=0}(a)$; call the intersection point of the two functions $\left(a^{*}, W^{*}\right)$.

Now consider the dynamics of $a$, determined by equation (16). When $\mu=0$ (in particular, at the optimal initial choice of $W$ ), the term on the left-hand side, $2 c^{\prime \prime}(a(t))+a(t) c^{\prime \prime \prime}(a(t))$, is non-negative (using assumption 1). The right-hand side is equal to $-\left(r_{A} W-\left(a(t) c^{\prime}(a(t))-c(a(t))\right)+a^{2} c^{\prime \prime}(a)\right)$, which by assumption 1 is negative for all non-negative values of $a$ and $W$. Hence $\dot{a} \leq 0$ at the optimal initial choice of $W$.

The function defined by $\dot{a}=0$ is

$$
W_{\dot{a}=0}(a) \equiv \frac{1}{r_{A}}\left(a c^{\prime}(a)-c(a)-a c^{\prime \prime}(a)\left(a+\left(r_{P}-r_{A}\right) \frac{\mu}{\mu+1}\right)\right) .
$$

Note that when $\mu=0$,

$$
W_{\dot{a}=0}(a) \equiv \frac{a c^{\prime}(a)-c(a)-a^{2} c^{\prime \prime}(a)}{r_{A}} \leq 0
$$

from assumption 1. Hence the function $W_{\dot{a}=0}(a)$ crosses the function $W_{\mu=0}(a)$ at a point below the horizontal axis. If $r_{P}=r_{A}$, then $W_{\dot{a}=0}(a) \leq 0$ for all $a \in[0, \hat{a}]$. If $r_{P}<r_{A}$, then $W_{\dot{a}=0}(a)>W_{\dot{W}=0}(a)$ for sufficiently small $a$. Since $W_{\dot{a}=0}(a)$ is continuous in $a$, the $W_{\dot{a}=0}(a)$ curve must therefore cross the $W_{\dot{W}=0}(a)$ at a value of $a$ that is strictly less than $a^{*}$.

We can now determine the dynamics of $a$ and $W$. The region of particular interest for the analysis is defined as follows. Let $\mathcal{W}(a) \equiv\left\{W \in \mathbb{R}_{+} \mid W \leq\right.$ $W_{\dot{W}=0}(a)$ and $W \leq W_{\mu=0}(a)$ and $\left.W \geq W_{\dot{a}=0}(a)\right\}$ for $a \in[0, \hat{a}]$. Let $\mathcal{D} \equiv$ $\bigcup_{a \in[0, \hat{a}]} \mathcal{W}(a)$. (The region $\mathcal{D}$ is illustrated as the shaded regions in figures 2 and 3.) For $(a, W) \in \mathcal{D}$, both $\dot{a}$ and $\dot{W}$ are non-positive. If $r_{P}=r_{A}$, then $\mathcal{D}$ is defined as the (lower) area between the curves $W_{\dot{W}=0}(a)$ and $W_{\mu=0}(a)$. If $r_{P}<r_{A}$, then $\mathcal{D}$ is further defined by the curve $W_{\dot{a}=0}(a)$.

An initial choice of $W$ on the portion of the $W_{\mu=0}(a)$ curve above the $W_{\dot{W}=0}(a)$ cannot be optimal. The reason is that the dynamics from this point involve $\dot{W} \geq 0$. Hence any path from such a point cannot converge to a steady state, and by transversality cannot be optimal.

Hence the optimal initial choice of $W$ must lie on the portion of the $W_{V^{\prime}=0}(a)$ curve below the $W_{\dot{W}=0}(a)$. (Note that this must involve an initial effort level 
less than $\hat{a}$, and hence less than the myopic level $a^{M}$.) The initial dynamics from such a point are $\dot{W} \leq 0$ and $\dot{a} \leq 0$. The resulting path lies in the region $\mathcal{D}$, and hence $\dot{W} \leq 0$ and $\dot{a} \leq 0$ along all parts of an optimal path. The dynamics of $w(t)$ then follows from equation (8).

If $r_{P}=r_{A}$, then assumption 1 ensures that the function $W_{\dot{a}=0}(a)$ is negative for all values of $a \in[0, \hat{a}]$. Hence the only feasible steady state is $a=W=0$. If $r_{P}<r_{A}$, then there is a second steady state with strictly positive $a \in\left(0, a^{*}\right)$ and $W \in\left(0, W^{*}\right)$; the optimal path converges to this steady state. (Note that the system cannot converge to a point on the horizontal axis. At such a point, the agent's effort is strictly positive but his value is 0 . But this cannot be optimal for the agent, who could generate a strictly positive value by reducing his effort.)

\section{Proof of Proposition 5}

The Proposition is immediate when the agent is relatively impatient $\left(r_{P}<r_{A}\right)$, since his equilibrium effort in this case is always strictly positive. To prove the Proposition when the agent is equally patient $\left(r_{P}=r_{A}\right)$, we consider the behaviour of the system for small values of $a$ and $W$.

From equation (A.24), as $(a, W) \rightarrow(0,0)$,

$$
\mu \rightarrow \frac{r_{P}\left(v-W-c^{\prime}(a)\right)-\left(a+r_{P}\right) a c^{\prime \prime}(a)}{r_{A} W+\left(a+r_{P}\right) a c^{\prime \prime}(a)}
$$

and so becomes unbounded as the system approaches the steady state. Using this fact, the dynamics of the agent's effort, from equation (16), tends towards

$$
\begin{aligned}
& {\left[c^{\prime \prime}(a(t))+a(t) c^{\prime \prime \prime}(a(t))\right] \dot{a}(t)} \\
& \quad=-\left(r_{A} W-\left(a(t) c^{\prime}(a(t))-c(a(t))\right)+a^{2} c^{\prime \prime}(a)\right)+a^{2}(t) c^{\prime \prime}(a(t))\left(r_{P}-r_{A}\right) .
\end{aligned}
$$

The expression in the square brackets on the left-hand side of this equation is of order (big-O) $c^{\prime \prime}(a)$ in $a$. The right-hand side of the equation is of order (big-O) $c(a)$ in $a$ and is linear in $W$.

Next, let the path followed by $W$ in $(a, W)$ space be denoted $W(a)$. Given the dynamics described by $\dot{a}$ and $\dot{W}$, this path is smooth. Consider a Taylor expansion of this path close to the steady state $(0,0): W(a)=W(0)+a W^{\prime}(0)+$ $a^{2} W^{\prime \prime}(0)+\ldots$ Obviously $W(0)=0$; and since $W(a) \geq 0$ for all $a$, it must therefore be that $W^{\prime}(0) \geq 0$. But since $\dot{W} \leq 0$ (proposition 4 ), it must also be that

$$
W(a) \leq W_{\dot{W}=0}(a) \equiv \frac{a c^{\prime}(a)-c(a)}{r_{A}} .
$$

Therefore $W^{\prime}(0) \leq W_{\dot{W}=0}^{\prime}(a)=\left.\left(a c^{\prime \prime}(a) / r_{A}\right)\right|_{a=0}=0$. Therefore $W^{\prime}(0)=0$, and the Taylor expansion of $W(a)$ becomes $W(a)=a^{2} W^{\prime \prime}(0)+\ldots$. That is, on the optimal path, $W(a)=O\left(a^{2}\right)$. 
Using this fact in equation (A.25), we see that the left-hand side is of order (big-O) $c^{\prime \prime}(a)$ in $a$, while the right-hand side is of order (big-O) $a^{2}$. Let $\beta(a) \equiv$ $a^{2} / c^{\prime \prime}(a)$ for $a>0$. (For example, with quadratic costs $c(a)=\gamma a^{2}$ with $\gamma>0$, $\beta(a)=a^{2} / 2 \gamma$.) Hence $\dot{a}=O(\beta(a))$ as $(a, W) \rightarrow(0,0)$.

Whether the integral $\int_{0}^{\infty} a(t) d t$ of the agent's effort is bounded above by a finite constant then depends on the order of $\beta(a)$. It is straightforward to show that if $\beta(a)$ is of order $a^{2}$ or higher, then the rate of decrease is sufficiently slow that the integral is unbounded. But if $\beta(a)$ is of lower order than $a^{2}$, then the rate of decrease is such that the integral is finite.

\section{Proof of Proposition 6}

We start by showing that in any stationary equilibrium $\mathbf{w}^{f}\left(h^{t}\right)=w^{f}=0$ for all $h^{t}$. Suppose not i.e., $w^{f}>0$. If $w^{s} \leq w^{f}$, then the optimal effort choice is 0 and the equilibrium payoff to the principal is negative and setting $w^{f}\left(h^{t}\right)=w^{s}=0$ is a profitable deviation after any $h^{t}$. Suppose next that $w^{s}>w^{f}>0$. A deviation to $\widehat{w}^{s}\left(h^{t}\right)=w^{s}-w^{f}, \widehat{w}^{f}\left(h^{t}\right)=0$ leaves the incentives for optimal effort choice in period $t$ unchanged since by assumption the future wage offers by the principal do not depend on the current ones in a stationary equilibrium. This deviation reduces the wage payments of the principal by $w^{f}>0$ and hence is clearly profitable.

We compute next a wage offer $w^{s}=w^{S R}>0$ and an effort choice function $a$ that constitute a stationary equilibrium for our game. We start by computing the equilibrium expected payoff $W^{S R}$ to the agent. By standard arguments, we have for $\Delta \rightarrow 0$ :

$$
W^{S R}=\max _{a} \frac{a w^{S R}-c(a)}{r_{A}+a} .
$$

By solving the first-order condition for $a$ and substituting, we get $r_{A} W^{S R}=$ $a^{S R} c^{\prime}\left(a^{S R}\right)-c\left(a^{S R}\right)$, where $a^{S R}$ solves problem (A.26.) Therefore we have after substituting:

$$
w^{S R}=c^{\prime}\left(a^{S R}\right)+\frac{a^{S R} c^{\prime}\left(a^{S R}\right)-c\left(a^{S R}\right)}{r_{A}} .
$$

Consider next a current (possibly deviating) wage offer of $w^{s}\left(h^{t}\right)=w, w^{f}\left(h^{t}\right)=$ $0 .{ }^{15}$ The agent's best response to this offer is given by a solution to the problem $\max _{a}\left\{a w \Delta-c(a) \Delta+\left(1-r_{A} \Delta\right)(1-a \Delta) W^{S R}\right\}$. The first-order condition for the problem is given by $\left(w-W^{S R}\right)=c^{\prime}(a)$. Denote the solution to this problem by $a(w, 0)$. By the implicit function theorem,

$$
\frac{d a}{d w}=\frac{1}{c^{\prime \prime}(a(w, 0))}
$$

\footnotetext{
${ }^{15} \mathrm{By}$ the same argument as above, any deviation where $w_{t}^{f}>0$ is dominated by a deviation where $w_{t}^{f}=0$, but with $w^{s}$ set so that incentives are unchanged.
} 
The expected payoff to the principal from this wage offer is

$$
a(w, 0) \Delta(v-w)+\left(1-r_{P} \Delta\right)(1-a(w, 0) \Delta) V^{S R},
$$

where $V^{S R}$ is the expected payoff to the principal along the path where $w_{t}^{s}=$ $w^{S R}>0$ for all $t$. For $\Delta \rightarrow 0$, the first-order condition for this problem is $\left(v-w-V^{S R}\right) \frac{d a}{d w}-a(w, 0)=0$. Substituting from equation (A.28), we have $\left(v-w-V^{S R}\right)-a(w, 0) c^{\prime \prime}(a(w, 0))=0$. At a stationary equilibrium, we must have the first order condition satisfied at $w=w^{S R}$. Therefore

$$
w^{S R}=v-\frac{a^{S R}\left(a^{S R}+r_{P}\right)}{r_{P}} c^{\prime \prime}\left(a^{S R}\right) .
$$

In a stationary equilibrium, equations (A.27) and (A.30) must hold simultaneously. By our assumptions, the former gives $w^{S R}$ as an increasing function of $a^{S R}$ while the latter gives a decreasing function. At $a^{S R}=0$, the former function starts above the latter, while in the limit as $a^{S R} \rightarrow \infty$, the latter decreases without bound. Therefore the values of these functions coincide at exactly one point.

\section{Proof of Proposition 7}

\section{Preliminaries}

Even though the stage game payoffs are not necessarily bounded, it is easy to see that for every $\Delta$, there is an $M(\Delta)$ such that $w_{t}^{f}>M(\Delta)$ and $w_{t}^{s}>M(\Delta)$ are strictly dominated. Hence it is without loss of generality to consider $w_{t} \in$ $[0, M(\Delta)] \times[0, M(\Delta)]$. Similarly, due to the convexity of the cost function, there is an $N(\Delta)$ such that $a>N(\Delta)$ is strictly dominated (given that wages are bounded). Under these natural restrictions, the set of paths $\left\{\left(w_{t}, a_{t}\right)\right\}_{t=0}^{\infty}$ is compact in the product topology.

Let $\mathcal{U}$ denote the set of equilibrium payoff vectors in the game i.e., $\mathcal{U}=$ $\{(V(\mathbf{w}, \mathbf{a}), W(\mathbf{w}, \mathbf{a})) \mid(\mathbf{w}, \mathbf{a}) \in \mathcal{E}\}$. Arguments similar to Abreu (1988) establish that $V$ and $W$ are continuous in the product topology. Hence there exists an equilibrium $(\underline{\mathbf{w}}, \underline{\mathbf{a}}) \in \mathcal{E}$ such that $V(\underline{\mathbf{w}}, \underline{\mathbf{a}})=\min _{(\mathbf{w}, \mathbf{a}) \in \mathcal{E}} V(\mathbf{w}, \mathbf{a}):=\underline{V}$.

Our goal is to show that, for any history $h^{t}, \underline{w}=\left(\underline{w}^{s}, \underline{w}^{f}\right)=\left(w^{S R}, 0\right)$ and $\underline{a}=a\left(w_{t}, W^{S R}\right)$, where $a\left(w_{t}, W^{S R}\right)$ is the best response by the agent if the current wage offer is $w_{t}$ and all future wage offers are $w\left(h^{s}\right)=w^{S R}$ for all $s>t$ (and hence $W^{S R}$ denotes the continuation payoff to the agent along this path of offers).

\section{Minimal continuation payoffs for given $w_{t}$}

Since all continuation games are strategically equivalent, the set of sequential equilibria at time $t, \mathcal{E}$, is the same as the set at $t+1$. Hence, since the agent's own action is unobservable, his optimal choice of effort in period $t$ depends only 
on the current offer $w_{t}$ and the continuation payoff $W(\mathbf{w}, \mathbf{a})$ induced by the continuation equilibrium $(\mathbf{w}, \mathbf{a}) \in \mathcal{E}$. For an arbitrary $w_{t}$, we let $a\left(w_{t}, W(\mathbf{w}, \mathbf{a})\right)$ denote this best response. We can find the principal's worst sequentially rational continuation equilibrium given the current offer $w_{t}=\left(w_{t}^{s}, w_{t}^{f}\right)$ by solving

$$
\begin{aligned}
\min _{(\mathbf{w}, \mathbf{a}) \in \mathcal{E}} a\left(w_{t}, W(\mathbf{w}, \mathbf{a})\right) \Delta\left(v-w_{t}^{s}\right) & -\left(1-a\left(w_{t}, W(\mathbf{w}, \mathbf{a})\right) \Delta\right) w_{t}^{f} \\
+ & \left(1-a\left(w_{t}, W(\mathbf{w}, \mathbf{a})\right) \Delta\right)\left(1-r_{P} \Delta\right) V(\mathbf{w}, \mathbf{a}) .
\end{aligned}
$$

Let $\left(\underline{\mathbf{w}}^{w_{t}}, \underline{\mathbf{a}}^{w_{t}}\right)$ be a solution to this problem ${ }^{16}$ and let $\underline{V}\left(w_{t}\right)$ be the minimized value as a function of the current wage offer $w_{t}$.

With these continuation strategies, it is clear that a current offer $w_{t}^{*}$ solving $w_{t}^{*} \in \arg \max _{w_{t}} \underline{V}\left(w_{t}\right)$, together with the continuation equilibrium $\mathbf{w}^{*}=\left(\underline{\mathbf{w}}^{w_{t}^{*}}\right)$, constitute a sequential equilibrium in which $\underline{V}\left(w_{t}^{*}\right)=\underline{V}$.

\section{Optimal penal codes}

Let $\left\{w_{t}\right\}_{t=0}^{\infty}$ denote the equilibrium path of wage offers in an arbitrary sequential equilibrium of the game. Let $\left\{a_{t}\right\}_{t=0}^{\infty}$ denote the best response of the agent to these wages. The following Lemma contains a simple characterization of wage paths that can be sustained in sequential equilibrium.

Lemma 3 A sequence $\left\{\widehat{w}_{t}\right\}_{t=0}^{\infty}$ of wage offers by the principal can be supported as an equilibrium path of a sequential equilibrium in the contracting game if and only if for all $t$,

$$
\sum_{i=t}^{\infty}\left(1-r_{P} \Delta\right)^{(i-t)}\left(\widehat{a}_{i} \Delta\left(v-\widehat{w}_{i}^{s}\right)-\left(1-\widehat{a}_{i} \Delta\right) \widehat{w}_{i}^{f}\right) \geq \underline{V},
$$

where $\left\{\widehat{a}_{t}\right\}_{t=0}^{\infty}$ is a best response to $\left\{\widehat{w}_{t}\right\}_{t=0}^{\infty}$.

Proof. (Only if.) If the inequality is violated, the continuation play following $t$ is not a sequential equilibrium by definition of $\underline{V}$.

(If.) Consider the following strategies for the two players:

$$
\begin{aligned}
\mathbf{w}\left(h^{t}\right) & =\widehat{w}_{t} & \text { if } h^{t} & =\left(\widehat{w}_{0}, \ldots, \widehat{w}_{t-1}\right), \\
\mathbf{w}\left(h^{t}\right) & =\underline{\mathbf{w}}^{w_{0}}\left(h^{t}\right) & \text { if } h^{t} & \neq\left(\widehat{w}_{0}, \ldots, \widehat{w}_{t-1}\right), \\
\mathbf{a}\left(h^{t}, w_{t}\right) & =\widehat{a}_{t} & \text { if }\left(h^{t}, w_{t}\right) & =\left(\widehat{w}_{0}, \ldots, \widehat{w}_{t-1}, \widehat{w}_{t}\right), \\
\mathbf{a}\left(h^{t}, w_{t}\right) & =\underline{\mathbf{a}}^{w_{0}}\left(h^{t}, w_{t}\right) & \text { if }\left(h^{t}, w_{t}\right) & \neq\left(\widehat{w}_{0}, \ldots, \widehat{w}_{t-1}, \widehat{w}_{t}\right) .
\end{aligned}
$$

Since $\left(\underline{\mathbf{w}}^{w_{0}}, \underline{\mathbf{a}}^{w_{0}}\right) \in \mathcal{E}$, the continuation play forms a sequential equilibrium in the continuation game after all possible deviations. Furthermore, by construction, the payoff to the principal is bounded from above by $\underline{V}$ after any deviation. If inequality (A.31) holds at all $t$, no profitable deviation is profitable, and the above strategies constitute a sequential equilibrium.

\footnotetext{
${ }^{16}$ Again the existence of a minimizer is given by the compactness of the set of equilibrium paths and the continuity of the payoffs.
} 


\section{Identifying continuation equilibrium profiles for payoff-minimizing equi- libria}

The remainder of the argument shows that we can take $\underline{\mathbf{w}}^{w_{t}}=\underline{\mathbf{w}}$ after all deviating wage offers $w_{t}$. This is the key step that shows that the worst punishment can be taken to be stationary. Let $\underline{\mathfrak{w}}:=\{\mathbf{w} \mid(\mathbf{w}, \mathbf{a}) \in \mathcal{E}$ for some $\mathbf{a}$, and $V(\mathbf{w}, \mathbf{a})=\underline{V}\}$. Notice that since $(\mathbf{w}, \mathbf{a}) \in \mathcal{E}, \mathbf{a}$ must be a best response to $\mathbf{w}$ i.e., $\mathbf{a}=B R(\mathbf{w})$. Furthermore, let $\mathfrak{w}^{\text {dev }}:=\arg \max _{\mathbf{w} \in \mathfrak{w}} W(\mathbf{w}, B R(\mathbf{w}))$, i.e., any $\mathbf{w}^{\text {dev }} \in \mathfrak{w}^{\text {dev }}$ induces the highest payoff to the agent amongst the equilibrium continuation wage offer strategies that keep the principal to expected payoff $\underline{V}$. Since both players' payoffs coincide for all continuation equilibria where the principal's strategy $\mathbf{w} \in \mathfrak{w}^{d e v}$, there is no loss of generality in restricting attention to an arbitrary (fixed) representative $\mathbf{w}^{\text {dev }}$ from $\mathfrak{w}^{\text {dev }}$. Let $\mathbf{a}^{\text {dev }}\left(w_{t}\right)$ denote the best response of the agent to $\left(w_{t}, \mathbf{w}^{d e v}\right)$ i.e., a deviation to $w_{t}$ in period $t$ followed by a continuation wage strategy $\mathbf{w}^{d e v}$. Also, define $w^{d e v} \in \arg \max _{w_{t}} V\left(\left(w_{t}, \mathbf{w}^{\operatorname{dev}}\right), \mathbf{a}^{\operatorname{dev}}\left(w_{t}\right)\right)$, where

$$
\begin{aligned}
V\left(\left(w_{t}, \mathbf{w}^{d e v}\right), \mathbf{a}^{\operatorname{dev}}\left(w_{t}\right)\right):= & a^{\operatorname{dev}}\left(w_{t}\right) \Delta\left(v-w_{t}^{s}\right)-\left(1-a^{d e v}\left(w_{t}\right) \Delta\right) w_{t}^{f} \\
& +\left(1-a^{\operatorname{dev}}\left(w_{t}\right) \Delta\right)\left(1-r_{P} \Delta\right) V\left(\mathbf{w}^{\operatorname{dev}}, \mathbf{a}^{d e v}\right) .
\end{aligned}
$$

We now show that for current wages close to $w^{\text {dev }}$, the worst continuation for the principal is induced by the continuation strategy $\mathbf{w}^{d e v}$. To show this, we contrast two equilibria: the principals worst sequentially rational continuation equilibrium given the current offer $w_{t}$, which we have denoted $\left(\underline{\mathbf{w}}^{w_{t}}, \underline{\mathbf{a}}^{w_{t}}\right)$; and the equilibrium $\left(\left(w_{t}, \mathbf{w}^{d e v}\right), \mathbf{a}^{\operatorname{dev}}\left(w_{t}\right)\right)$.

Lemma 4 There is $a \varepsilon>0$ and $\bar{\Delta}>0$ such that if $\left|w_{t}-w^{\text {dev }}\right|<\varepsilon$ and $\Delta<\bar{\Delta}$, then $\underline{V}\left(w_{t}\right)=V\left(\left(w_{t}, \mathbf{w}^{\text {dev }}\right), \mathbf{a}^{\text {dev }}\left(w_{t}\right)\right)$.

Proof. Observe first that for all $w_{t}$,

$$
\begin{aligned}
\underline{V}\left(w_{t}\right) \leq a^{\operatorname{dev}}\left(w_{t}\right) \Delta\left(v-w_{t}^{s}\right)-\left(1-a^{\operatorname{dev}}\left(w_{t}\right) \Delta\right) w_{t}^{f} \\
\\
+\left(1-a^{\operatorname{dev}}\left(w_{t}\right) \Delta\right)\left(1-r_{P} \Delta\right) V\left(\mathbf{w}^{\operatorname{dev}}, \mathbf{a}^{\operatorname{dev}}\right)
\end{aligned}
$$

by definition. Hence for all $\delta>0$, there is a $\bar{\Delta}>0$ such that for all $\Delta<\bar{\Delta}$, $\underline{V}\left(w_{t}\right) \leq V\left(\mathbf{w}^{d e v}, \mathbf{a}^{d e v}\right)+\delta$ using the fact that in equilibrium, wages are bounded.

From the definition of $w^{d e v}$, we know from the principal's first-order condition for $w_{t}^{s}$ that

$$
v-\left(w^{d e v, s}-w^{\operatorname{dev}, f}\right)-V\left(\mathbf{w}^{d e v}, \mathbf{a}^{d e v}\right)>0 .
$$

Here, we use the fact that an increase in $w_{t}^{s}$, with $w_{t}^{f}$ and all future wages fixed, must induce a greater current effort from the agent as a best response. Compare this to the inequality that must hold for the principal's payoff to be increasing in $a_{t}$ in the equilibrium $\left(\underline{\mathbf{w}}^{w_{t}}, \underline{\mathbf{a}}^{w_{t}}\right)$ :

$$
v-\left(w_{t}^{s}-w_{t}^{f}\right)-\underline{V}\left(w_{t}\right)>0 .
$$


From inequality (A.32), we can see that there is an $\varepsilon>0$ such that inequality (A.33) holds if $\left|w_{t}-w^{\text {dev }}\right|<\varepsilon$.

If the worst continuation equilibrium after $w_{t},\left(\underline{\mathbf{w}}^{w_{t}}, \underline{\mathbf{a}}^{w_{t}}\right) \notin \mathfrak{w}^{\text {dev }}$, then we can find another equilibrium with a lower payoff to the principal. To see this, note simply that if $\left(\underline{\mathbf{w}}^{w_{t}}, \underline{\mathbf{a}}^{w_{t}}\right) \notin \mathfrak{w}^{d e v}$, then either $V\left(\underline{\mathbf{w}}^{w_{t}}, \underline{\mathbf{a}}^{w_{t}}\right)>\underline{V}$ or $V\left(\underline{\mathbf{w}}^{w_{t}}, \underline{\mathbf{a}}^{w_{t}}\right)=$ $\underline{V}$ and $W\left(\underline{\mathbf{w}}^{w_{t}}, \underline{\mathbf{a}}^{w_{t}}\right)<W\left(\mathbf{w}^{d e v}, \mathbf{a}^{d e v}\right)$. In the first case, the principal's payoff can be reduced by making a small enough unconditional payment to the agent in the current period of the continuation game. This has no effect on the agent's optimal continuation strategies, maintains the agent's participation constraint and increases the agent's continuation value. But the agent's optimal current effort level $a\left(w_{t}, W\right)$ is strictly decreasing in $W$ if $a\left(w_{t}, W\right)>0$. Hence this unconditional payment reduces the agent's current effort, and so the principal's value.

In the second case, a switch to the continuation equilibrium $\left(\mathbf{w}^{d e v}, \mathbf{a}^{d e v}\right)$ increases the agent's continuation payoff without violating any incentive constraints, since $\left(\mathbf{w}^{d e v}, \mathbf{a}^{d e v}\right) \in \mathcal{E}$ by assumption. Hence the payoff to the principal is reduced in this case also. Furthermore by Lemma 3, the path with reduced wage payments can also be supported as a sequential equilibrium.

The previous Lemma establishes a lower bound to what the principal can guarantee, by choosing $w_{t}$ close to $w^{d e v}$. Hence the principal's lowest equilibrium payoff cannot be below that resulting from $\left(w^{d e v}, \mathbf{w}^{\text {dev }}\right)$ : a fact that we establish in the next lemma.

Lemma 5 In the continuous-time limit, the lowest payoff $\underline{V}$ to the payoff is given by $\underline{V}=V\left(\left(w^{\text {dev }}, \mathbf{w}^{\text {dev }}\right), \mathbf{a}^{\text {dev }}\left(w_{t}\right)\right)$.

Proof. Since by definition, $\underline{V} \geq \underline{V}\left(w_{t}\right)$ for all $w_{t}$, Lemma 4 implies that $\underline{V} \geq$ $V\left(\left(w_{t}, \mathbf{w}^{d e v}\right), \mathbf{a}^{\text {dev }}\left(w_{t}\right)\right)$. Since $\left(\mathbf{w}^{d e v}, \mathbf{a}^{\text {dev }}\right) \in \mathcal{E}, w\left(h^{t}\right)=w^{d e v}$ is a best response for the principal for all $h^{t}$, and therefore $\underline{V}=V\left(\left(w^{\operatorname{dev}}, \mathbf{w}^{\operatorname{dev}}\right), \mathbf{a}^{\operatorname{dev}}\left(w_{t}\right)\right)$.

\section{Proof of the main result}

The final step is to bring together these Lemmas, noting that the best current wage for the principal, given the equilibrium continuation path of wages $\mathbf{w}^{\text {dev }}$, is $w^{\text {dev }}$. Hence we have constructed a stationary equilibrium with payoff $\underline{V}$ to the principal; but since there is a unique stationary equilibrium, we can identify this equilibrium precisely.

Lemma 6 The payoff to the principal in the stationary equilibrium coincides with the worst equilibrium payoff for the principal: $\underline{V}=V^{S R}$.

Proof. By the previous Lemmas, we have $\underline{V}=V(\mathbf{w}, \mathbf{a})$, where $w_{t}=w^{\text {dev }} \forall h^{t}$ and $a\left(h^{t}, w_{t}\right)=a^{d e v}\left(w_{t}\right) \quad \forall h^{t}$. By the uniqueness of the stationary equilibrium, we conclude that $w^{d e v}=w^{S R}$ and $\mathbf{a}\left(h^{t}, w_{t}\right)=a\left(w_{t}, W^{S R}\right)$, and the claim follows. 


\section{Proof of Lemma 2}

The proof has two parts. First, consider two continuation games in which $(V(T), W(T))=\left(V^{S R}, W\right)$ and $(V(T), W(T))=\left(V^{S R}, W^{\prime}\right)$ with $W<W^{\prime}$. We claim that the principal's payoff is higher in the first game. Take the best (from the principal's point of view) equilibrium wage sequence of the second continuation game with $W(T)=W^{\prime}$. If the wages $w(t)$ for $t \leq T$ are the same in the first continuation game, then the agent's continuation payoff $W(t)$ in the first continuation game must be lower than in the second, for all $t \leq T$. But then the agent's equilibrium action $a(t)$ in the first continuation game is higher than in the second, for all $t \leq T$, and so the principal's payoff is also higher. Hence $V(t)>V^{S R}$. And by Lemma 3 in this Appendix, these wages can be supported in a sequentially rational equilibrium.

The second part of the proof shows that $V(\underline{W})=V^{S R}$. Since $\underline{W}$ is a sequentially rational continuation payoff for the agent, we know that $V(\underline{W}) \geq V^{S R}$. If $V(\underline{W})>V^{S R}$, then consider the modified wage sequence where the principal offers $w_{t}=0$ for initial period of length $\varepsilon$ and then reverts to the equilibrium path associated with payoffs $(V(\underline{W}), \underline{W})$. By continuity, the new path also induces a payoff to the principal above $V^{S R}$ and hence can be supported in a sequentially rational equilibrium, by Lemma 3. But the agent gets a lower equilibrium payoff, contradicting the assumption that $\underline{W}$ is the lowest payoff to the agent.

\section{Proof of Proposition 9}

Denote the initial value to the principal in the best WRP equilibrium by $V_{0}$. Suppose that $V_{t}<V_{0}$ for some $t$ in that equilibrium, in the discrete-time version of the game with period length $\Delta$. Let $\tau$ be $\min \left\{t \mid V_{t}>V_{t+1}\right\}$. By definition, $V_{\tau}=V_{0}$; and by the definition of a WRP equilibrium, $W_{\tau+1}>W_{\tau}$.

But then, since the best response of the agent $a\left(w_{t}, W_{t+1}\right)$ is decreasing in $W_{t+1}$, we can construct a sequentially rational equilibrium payoff $\left(V^{\prime}, W^{\prime}\right)$ with continuation payoffs $\left(V_{\tau}, W_{\tau}\right)$ such that $V^{\prime}>V_{\tau}$ and $W^{\prime}<W_{\tau}$, contradicting the assumption that $V_{0}=V_{\tau}$ is the highest WRP payoff to the principal.

\section{References}

Dilip Abreu. On the theory of infinitely repeated games with discounting. Econometrica, 56(2):383-396, 1988.

Dirk Bergemann and Ulrich Hege. Venture capital financing, moral hazard, and learning. Journal of Banking \& Finance, 22(6):703-735, 1998.

Dirk Bergemann and Ulrich Hege. The financing of innovation: learning and stopping. RAND Journal of Economics, 36(4):719-752, 2005. 
Bruno Biais, Thomas Mariotti, G Plantin, and JC Rochet. Dynamic security design: Convergence to continuous time and asset pricing implications. The Review of Economic Studies, 74:345-390, 2007.

Bruno Biais, Thomas Mariotti, Jean-Charles Rochet, and Stéphane Villeneuve. Large risks, limited liability, and dynamic moral hazard. Econometrica, 78(1): 73-118, 2010.

Joseph Farrell and Eric Maskin. Renegotiation in repeated games. Games and Economic Behavior, 1(4):327-360, 1989.

Hugo Hopenhayn and Juan Pablo Nicolini. Optimal unemployment insurance. Journal of Political Economy, 105:412-438, 1997.

Takashi Kamihigashi. Necessity of transversality conditions for infinite horizon problems. Econometrica, 69(4):995-1012, 2001.

Jean-Jacques Laffont and David Martimort. The Theory of Incentives: the Principal-Agent Model. Princeton University Press, 2002.

W. Bentley MacLeod and James M. Malcomson. Motivation and markets. American Economic Review, 1998.

James Malcomson and Francis Spinnewyn. The multi-period principal-agent problem. Review of Economic Studies, 55:391-408, 1988.

Christopher Phelan and Robert Townsend. Computing multi-period, information-constrained optima. Review of Economic Studies, 58:853-881, 1991.

Yuliy Sannikov. Games with imperfectly observable actions in continuous time. Econometrica, 75(5):1285-1329, 2007.

Yuliy Sannikov. A continuous-time version of the principal-agent problem. Review of Economic Studies, 75(3):957-984, 2008.

Carl Shapiro and Joseph Stiglitz. Equilibrium unemployment as a worker discipline device. The American Economic Review, 1984.

Stephen Shavell and Laurence Weiss. The optimal payment of unemployment insurance benefits over time. Journal of Political Economy, 87(6):1437-1362, 1979 .

Stephen Spear and Sanjay Srivastava. On repeated moral hazard with discounting. Review of Economic Studies, 54:599-617, 1987.

Stephen Thomas. Areva and edf: Business prospects and risks in nuclear energy. Technical report, PSIRU, 2009. 
Noah Willams. On dynamic principal-agent problems in continuous time. Available at http://www.princeton.edu/ noahw/pa1.pdf, December 2006. 